\title{
Modelling of Dynamic Damage and Failure in Aluminium Alloys
}

\author{
R. Vignjevic ${ }^{1}$, N. Djordjevic, J. Campbell, V. Panov \\ ${ }^{1}$ Department of Applied Mechanics, School of Engineering, \\ Cranfield University, Cranfield, Bedfordshire, \\ MK43 OAL, United Kingdom \\ e-mail: v.rade@cranfield.ac.uk
}

\begin{abstract}
A physically based damage and failure model, applicable to orthotropic metals is proposed in this paper. To account for the physical mechanisms of failure, the concept of thermally activated damage initially proposed by Klepaczko [1], has been adopted as the basis for the model. This assumption makes the proposed damage/failure model compatible with the Mechanical Threshold Strength (MTS) model [2-6], which was used within the overall constitutive model to describe material behaviour in the plastic regime. A shock equation of state [7] was coupled with the rest of the constitutive model to allow for modelling of shock wave propagation in the material. The new model was implemented in DYNA3D [8] and coupled with our in-house non-linear transient SPH code, MCM (Meshless Continuum Mechanics).

Parameters for the new constitutive model, i.e. parameters for the plasticity model and the damage model, were derived on the basis of the uniaxial tensile tests and Taylor anvil tests. The subject of investigation is a polycrystalline aluminium alloy AA7010, whose orthotropy is a consequence of meso-scale phase distribution, or grain morphology. Tensile tests were performed for the range of temperatures between $223.15 \mathrm{~K}$ and $473.15 \mathrm{~K}$, and strain rates between $6.4 \times 10^{-4} \mathrm{~s}^{-1}$ and $6.4 \times 10^{2} \mathrm{~s}^{-1}$.
\end{abstract}


In order to validate the new damage model, a numerical simulation of Taylor anvil tests has been performed for AA7010, using a single stage gas gun at velocity of $200 \mathrm{~m} / \mathrm{s}$. The numerical analysis clearly demonstrates the ability of this new model to predict experimentally observed damage and failure. 


\section{Introduction}

Accurate constitutive models for Aluminium alloys (AA) have been of significant interest to the Aerospace industry, as the ability to predict strain rate dependent mechanical behaviour of orthotropic AA, undergoing impact and dynamic loading, such as bird strike, debris impacts, tyre burst, etc. Therefore, the focus of this research is modelling of dynamic behaviour including for strain rate effects and shock wave propagation.

Prior to failure, ductile materials undergo significant plastic deformation, which has a major influence on damage evolution. These materials usually fail as the result of nucleation, growth and coalescence of micro-voids, resulting in the loss of the load carrying capacity of the material. Experimental observations show that plastic deformation and accumulation of micro-damage have a tendency to localise. The progressive physical process of degradation of the material mechanical properties up to complete failure is commonly referred to as the damage process.

These two distinct dissipative mechanical processes, i.e. plasticity and damage, are the main causes of non-linear material behaviour. A large number of multi-dissipative models for this type of behaviour for ductile metals have been proposed. Some include damage micromechanics (micromechanical damage models, e.g. [9-11]), whilst others are based upon Continuum Damage Theory (phenomenological damage models [12-16]).

The approach proposed in this paper for coupling plasticity and damage is based on the assumption that there are two separate damage and plasticity loading surfaces, with two independent associated flow rules. This type of starting assumption has been extensively used by many authors in the literature [17-20].

In this work, the physically based Mechanical Threshold Stress (MTS) model [2] was adopted to model evolution of the plastic deformation of aluminium alloy AA7010, including the 
strain rate and temperature effects. The MTS model is based on a thermally activated dislocation motion and thermal activation energy defined in terms of Arhhenius law [2]. The key feature of the model is that the strain rate and temperature effects are coupled in a way so that the model accurately predicts changes in the flow stress, which are typical for the most of FCC and BCC metals. A significant body of experimental data indicates that dynamic behaviour of these metals is characterised by increase in flow stress as a consequence of increase in loading rate (strain rate) for a given reference initial temperature. Furthermore, it was observed in a number of experiments that the similar effects can be induced by decreasing the initial temperature while keeping the rate of loading (strain rate) fixed, see for instance [21-24]. Consequently, the parameters for the MTS model for AA7010 were derived on the basis of tensile tests performed over a range of temperatures and strain rates, between $223.15 \mathrm{~K}$ and $473.15 \mathrm{~K}$, and $6.4 \times 10^{-4} \mathrm{~s}^{-1}$ and $6.4 \times 10^{1} \mathrm{~s}^{-1}$, respectively. Testing at the low temperatures allowed for model to be used for the range of strain rates of interest (from $10^{2} s^{-1}$ and $\left.10^{4} s^{-1}\right)$.

Taylor anvil test performed at velocity of $200 \mathrm{~m} / \mathrm{s}$ was used for material model validation. The specimen was a $9.3 \mathrm{~mm}$ diameter cylinder, with a length-to-diameter ratio $L / D=5$. Depending on the initial impact velocity and material strength, a wide range of strain rates, temperatures and strains can be achieved in a single test. Therefore, the Taylor anvil test provides a well-defined set of experimental data, which allows for validation of material models and their implementation into hydrocodes, such as DYNA3D.

The notation used throughout this paper is as follows: italic symbols denote the scalar variables (for instance temperature $\theta$, free energy $\psi$ ), bold face notation represents the second order tensors (stress and damage tensors $\boldsymbol{\sigma}$ and $\mathbf{D}$, respectively) and capital Euclid 
The paper comprises four sections. Section 2 introduces a framework of thermodynamics of the constitutive model, defines the thermodynamic (state and dissipation) potentials, which constitute the orthotropic elastic-plastic model with damage, and describes the experimental characterisation of material model parameters. The numerical validation of the proposed model is done through the Taylor anvil test, as described in the Section 3. The paper concludes with the summary and conclusions given in the Section 4. Parts of the derivation of the damage evolution model are given in the Appendix A and some simulation results for a Taylor test are given in the Appendix B.

\section{Anisotropic elastoplasticity with damage in the framework of thermodynamics}

In order to derive and couple different parts of the constitutive model, i.e. elastic, plastic and damage, the framework of irreversible thermodynamics, outlined in the following subsection, was used. For high loading rates, which are of interest in this research, one can assume that the local generation of heat results in adiabatic heating of the material.

\subsection{Thermodynamic framework with internal variables and thermodynamic potentials} The elastic-plastic-damage model is developed in the framework of thermodynamics with internal variables using a principle of equipresence $[25 ; 26]$, whereby the thermodynamic state potentials (Helmholtz free energy or internal energy) can be defined in terms of elastic strain, temperature (or entropy) and a set of internal variables $v$, which describe the microscopic dissipative processes. By assuming that each irreversible process is described by a pair of internal variables for isotropic and kinematic hardenings, Helmholtz free energy can be defined similarly to $[17 ; 27 ; 28]$ as:

$\psi=\psi\left(\boldsymbol{\varepsilon}_{e}, \theta, \alpha^{\prime}, \boldsymbol{\alpha}^{\prime \prime}, \mathbf{D}, \beta^{\prime}, \boldsymbol{\beta}^{\prime \prime}\right)$ 
where: $\boldsymbol{\varepsilon}_{e}$ is the elastic-strain tensor, $\theta$ is temperature, $\alpha^{\prime}$ is a hardening variable that describes the size of the yield surface (isotropic hardening), $\boldsymbol{\alpha}^{\prime \prime}$ is a hardening variable that describes a shift in the centre of the yield surface (kinematic hardening), D represents damage tensor and is a measure of degradation of material integrity, $\beta^{\prime}$ represents a damage variable describing the size of the damage surface and $\boldsymbol{\beta}^{\prime \prime}$ is a damage variable that describes the shift of the centre of the damage surface and is a tensor of the same order as $\mathbf{D}$.

Note that the plastic deformation and damage differ in terms of the effects they induce on the material compliance. The damage tensor $\mathbf{D}$ affects the material compliance and consequently is included in the list of arguments in (1). However, the plastic strain tensor is not a state variable and consequently was not used in the definition of thermodynamic potentials.

The principle of equipresence states that internal energy, stress, temperature and internal variables are all defined in terms of the same set of variables [25;26]. Consequently, all constitutive equations are expressed in terms of the set of arguments introduced in equation (1). However, if one assumes that the stress and specific heat are not the functions of hardening variables [29], and that hardenings of two irreversible processes are independent, the Helmholtz free energy can be additively decomposed as follows $[17 ; 27 ; 30]$ :

$$
\rho \psi\left(\boldsymbol{\varepsilon}_{e}, \theta, \alpha^{\prime}, \boldsymbol{\alpha}^{\prime \prime}, \mathbf{D}, \beta^{\prime}, \boldsymbol{\beta}^{\prime \prime}\right)=W\left(\boldsymbol{\varepsilon}_{e}, \theta, \mathbf{D}\right)+H_{p}\left(\theta, \alpha^{\prime}, \boldsymbol{\alpha}^{\prime \prime}\right)+H_{d}\left(\theta, \beta^{\prime}, \boldsymbol{\beta}^{\prime \prime}\right)
$$

where: $W\left(\boldsymbol{\varepsilon}_{e}, \theta, \mathbf{D}\right)$ is the stored elastic energy, $H_{p}\left(\theta, \alpha^{\prime}, \boldsymbol{\alpha}^{\prime \prime}\right)$ is the energy related to plastic hardening and $H_{d}\left(\theta, \beta^{\prime}, \boldsymbol{\beta}^{\prime \prime}\right)$ is the energy related to damage hardening.

By calculating a rate of change of free energy from the equation (2), one can obtain a constitutive equations for the stress, entropy and rate of internal dissipation $\Gamma$, from the principle laws of thermodynamics [31], as: 


$$
\begin{aligned}
\Gamma & =\boldsymbol{\sigma}: \mathbf{d}-\rho \psi-\rho \theta s \\
& =\left(\boldsymbol{\sigma}-\rho \frac{\partial W}{\partial \boldsymbol{\varepsilon}_{e}}\right): \boldsymbol{\varepsilon}-\left(s+\rho \frac{\partial \psi}{\partial \theta}\right) \theta \\
& +\rho \frac{\partial W}{\partial \boldsymbol{\varepsilon}_{e}}: \boldsymbol{\varepsilon}_{p}-\rho \frac{\partial H_{p}}{\partial \alpha^{\prime}} \alpha^{\prime}-\rho \frac{\partial H_{p}}{\partial \boldsymbol{\alpha}^{\prime \prime}}: \boldsymbol{\alpha}^{\prime \prime}-\rho \frac{\partial W}{\partial \mathbf{D}}: \mathbf{D}-\rho \frac{\partial H_{d}}{\partial \beta^{\prime}} \beta^{\prime}-\rho \frac{\partial H_{d}}{\partial \boldsymbol{\beta}^{\prime \prime}}: \boldsymbol{\beta}^{\prime \prime}
\end{aligned}
$$

where:

$$
s\left(\boldsymbol{\varepsilon}_{e}, \theta, \alpha^{\prime}, \boldsymbol{\alpha}^{\prime \prime}, \beta^{\prime}, \boldsymbol{\beta}^{\prime \prime}\right)=-\frac{\partial \psi}{\partial \theta} \quad \boldsymbol{\sigma}\left(\boldsymbol{\varepsilon}_{e}, \theta\right)=\rho \frac{\partial W}{\partial \boldsymbol{\varepsilon}_{e}}
$$

are the constitutive functions for entropy and Cauchy stress, respectively, derived from the second law of thermodynamics, which must hold for any rate of total deformation and temperature. Consequently, the rate of internal dissipation is:

$$
\begin{aligned}
\Gamma & =\rho \frac{\partial W}{\partial \boldsymbol{\varepsilon}_{e}}: \boldsymbol{\varepsilon}_{p}-\rho \frac{\partial H_{p}}{\partial \alpha^{\prime}} \alpha^{\prime}-\rho \frac{\partial H_{p}}{\partial \boldsymbol{\alpha}^{\prime \prime}}: \boldsymbol{\alpha}^{\prime \prime}-\rho \frac{\partial W}{\partial \mathbf{D}}: \mathbf{D}-\rho \frac{\partial H_{d}}{\partial \beta^{\prime}} \beta^{\prime}-\rho \frac{\partial H_{d}}{\partial \boldsymbol{\beta}^{\prime \prime}}: \boldsymbol{\beta}^{\prime \prime}= \\
& =\boldsymbol{\sigma}: \boldsymbol{\varepsilon}_{p}+R^{\prime} \alpha^{\prime}+\mathbf{R}^{\prime \prime}: \boldsymbol{\alpha}^{\prime \prime}+\mathbf{Y}: \mathbf{D}+Y^{\prime} \beta^{\prime}+\mathbf{Y}^{\prime \prime}: \boldsymbol{\beta}^{\prime \prime}=\Gamma_{p}+\Gamma_{d}
\end{aligned}
$$

where the conjugate thermodynamic forces are:

$$
\begin{aligned}
& R^{\prime} \equiv-\rho \frac{\partial H_{p}}{\partial \alpha^{\prime}} \\
& \mathbf{R}^{\prime \prime} \equiv-\rho \frac{\partial H_{p}}{\partial \boldsymbol{\alpha}^{\prime \prime}} \\
& \mathbf{Y} \equiv-\rho \frac{\partial W}{\partial \mathbf{D}} \\
& \mathrm{Y}^{\prime} \equiv-\rho \frac{\partial H_{d}}{\partial \beta^{\prime}} \\
& \mathbf{Y}^{\prime \prime} \equiv-\rho \frac{\partial H_{d}}{\partial \boldsymbol{\beta}^{\prime \prime}}
\end{aligned}
$$

A free energy function (2) can be further specified in terms of elastic energy and hardening components as in [30]:

$$
\begin{gathered}
\rho \psi\left(\boldsymbol{\varepsilon}_{e}, \theta, \alpha^{\prime}, \boldsymbol{\alpha}^{\prime \prime}, \mathbf{D}, \beta^{\prime}, \boldsymbol{\beta}^{\prime \prime}\right)=\frac{1}{2} \boldsymbol{\varepsilon}_{e}: \quad(\theta, \mathbf{D}): \boldsymbol{\varepsilon}_{e}-\gamma\left(\boldsymbol{\varepsilon}_{e}\right): \boldsymbol{\varepsilon}_{e}\left(\theta-\theta_{0}\right)-c \theta \ln \left(\frac{\theta}{\theta_{0}}\right) \\
+H_{p}\left(\theta, \alpha^{\prime}, \boldsymbol{\alpha}^{\prime \prime}\right)+H_{d}\left(\theta, \beta^{\prime}, \boldsymbol{\beta}^{\prime \prime}\right)
\end{gathered}
$$

where $(\mathbf{D}, \theta)$ is a material stiffness tensor, $\gamma\left(\boldsymbol{\varepsilon}_{e}\right)=\frac{\partial \boldsymbol{\sigma}}{\partial \theta}$ is a thermodynamic force conjugate to the tensor of thermal expansion and $c$ is specific heat, which is a material property defined per unit volume.

Note that the elastic properties of a material depend on temperature and the accumulated damage D. The plastic deformation and damage dissipation processes are not directly 
dependent on temperature. Temperature increase due to adiabatic dissipation in the proposed model is calculated using Bammann's approach, see for instance [32; 33].

Based on the assumption that energy dissipations due to plastic flow and damage processes are independent, the criterion for the maximum total dissipation rate can be expressed in terms of objective function and two pseudo potentials:

$L=\boldsymbol{\sigma}: \boldsymbol{\varepsilon}_{p}+R^{\prime} \alpha^{\prime}+\mathbf{R}^{\prime \prime}: \boldsymbol{\alpha}^{\prime \prime}+\mathbf{Y}: \mathbf{D}+\mathrm{Y}^{\prime} \beta^{\prime}+\mathbf{Y}^{\prime \prime}: \boldsymbol{\beta}^{\prime \prime}-\lambda_{p} F_{p}-\lambda_{d} F_{d}$

where $F_{p}\left(\boldsymbol{\sigma}, R^{\prime}, \mathbf{R}^{\prime \prime}\right)=0$ and $F_{d}\left(\mathbf{Y}, \mathbf{Y}^{\prime}, \mathbf{Y}^{\prime \prime}\right)=0$ are, respectively, plastic and damage potentials of the associated flaw laws; $\lambda_{p}$ and $\lambda_{d}$ are Lagrange multipliers for the plastic and damage dissipation. The maximum dissipation is mathematically determined by an extreme values of the objective function (8), defined in the space of thermodynamic conjugate forces as:

$$
\begin{array}{lll}
\frac{\partial L}{\partial \mathbf{\sigma}}=0 & \frac{\partial L}{\partial R^{\prime}}=0 & \frac{\partial L}{\partial \mathbf{R}^{\prime \prime}}=0 \\
\frac{\partial L}{\partial \mathbf{Y}}=0 & \frac{\partial L}{\partial Y^{\prime}}=0 & \frac{\partial L}{\partial \mathbf{Y}^{\prime \prime}}=0
\end{array}
$$

which respectively lead to the following evolution equations:

$$
\begin{array}{lll}
\boldsymbol{\varepsilon}_{p}=\lambda_{p} \frac{\partial F_{p}}{\partial \boldsymbol{\sigma}} & \alpha^{\prime}=\lambda_{p} \frac{\partial F_{p}}{\partial R^{\prime}} & \boldsymbol{\alpha}^{\prime \prime}=\lambda_{p} \frac{\partial F_{p}}{\partial \mathbf{R}^{\prime \prime}} \\
\mathbf{D}=\lambda_{d} \frac{\partial F_{d}}{\partial \mathbf{Y}} & \beta^{\prime}=\lambda_{d} \frac{\partial L}{\partial Y^{\prime}} & \boldsymbol{\beta}^{\prime \prime}=\lambda_{d} \frac{\partial L}{\partial \mathbf{Y}^{\prime \prime}}
\end{array}
$$

The plastic and damage potentials satisfy the Hertz - Signori - Moreau (Kuhn Tucker) conditions:
$F_{p} \leq 0$
$\lambda_{p} \geq 0$
$\lambda_{p} F_{p}=0$
$F_{d} \leq 0$
$\lambda_{d} \geq 0$
$\lambda_{d} F_{d}=0$

for which the Lagrange multipliers are defined using the consistency conditions. The functional forms of dissipation potentials are given in subsection 2.2, following definition of damage effect tensor given in the remainder of this subsection. 


\subsubsection{Effective stress, damage effect tensor and damage energy release rate}

Damage in the proposed material model, is defined by using the principle of strain energy equivalence, originally derived by Cordebois and Sidoroff [34], as a generalization of pioneering work of Kachanov [35]. Kachanov developed a representation of an average effect of distributed microcracks by using effective stress which "acts" on the virgin material as oppose to the nominal stress which "acts" on damaged material. A linear relationship between the effective and nominal Cauchy stress, which was originally defined for one dimensional problem, is now expressed in terms of a damage effect tensor, which is suitable for modelling of anisotropic damage:

$\boldsymbol{\sigma}=(\mathbf{D}): \boldsymbol{\sigma}$

where $\boldsymbol{\sigma}$ denotes effective Cauchy stress and $(\mathbf{D})$ is a fourth order damage effect tensor, which is a function of a second order damage tensor $\mathbf{D}$.

The energy equivalence principle states that the complementary elastic energy for a damaged material is the same as that of a fictitious undamaged material:

$\frac{1}{2} \boldsymbol{\sigma}^{\mathrm{T}}:: \boldsymbol{\sigma}=\frac{1}{2} \boldsymbol{\sigma}^{\mathrm{T}}:: \boldsymbol{\sigma}$

where and are material compliance tensors for damaged and virgin (undamaged) material, respectively. By substituting $\boldsymbol{\sigma}=(\mathbf{D}): \boldsymbol{\sigma}$ into the above equation, the resulting expression provides the relationship between the compliance (consequently stiffness tensors and ) of damaged and undamaged material as:
$={ }^{T}(\mathbf{D}):$
$=-T(\mathbf{D}):$

To maintain the symmetry of the effective stress tensor, damage effect tensor is defined by a product type symmetrisation, see for instance $[12 ; 36]$, in the following form:

$$
(\mathbf{D})=(\mathbf{I}-\mathbf{D})^{-1 / 2}(\mathbf{I}-\mathbf{D})^{-1 / 2} \quad{ }_{i j k l}=\left(\delta_{i k}-\mathbf{D}_{i k}\right)^{-1 / 2}\left(\delta_{j l}-\mathbf{D}_{j l}\right)^{-1 / 2}
$$

and for the principle directions of damage, the tensor is given in the matrix form as: 


$$
(\mathbf{D})=\left[\begin{array}{cccccc}
\frac{1}{1-D_{1}} & 0 & 0 & 0 & 0 & 0 \\
0 & \frac{1}{1-D_{2}} & 0 & 0 & 0 & 0 \\
0 & 0 & \frac{1}{1-D_{3}} & 0 & 0 & 0 \\
0 & 0 & 0 & \frac{1}{\sqrt{\left(1-D_{2}\right)\left(1-D_{3}\right)}} & 0 & 0 \\
0 & 0 & 0 & 0 & \frac{1}{\sqrt{\left(1-D_{1}\right)\left(1-D_{3}\right)}} & 1 \\
0 & 0 & 0 & 0 & 0 & \frac{1}{\sqrt{\left(1-D_{1}\right)\left(1-D_{2}\right)}}
\end{array}\right]
$$

where the $D_{i}, i=1,2,3$ are principle values of the damage tensor and $0 \leq D_{i} \leq 1$. In this definition, $D_{i}=0$ corresponds to a virgin material, while $D_{i}=1$ corresponds to a fully damaged material. Damage effect tensor (16) and (17) can now be substituted in the Equation (15), leading to the stiffness tensor of a damaged material:

$$
=\left[\begin{array}{cccccc}
11\left(1-D_{1}\right)^{2} & 12\left(1-D_{1}\right)\left(1-D_{2}\right) & 31\left(1-D_{3}\right)\left(1-D_{1}\right) & 0 & 0 & 0 \\
& 22\left(1-D_{2}\right)^{2} & 23\left(1-D_{2}\right)\left(1-D_{3}\right) & 0 & 0 & 0 \\
& & 33\left(1-D_{3}\right)^{2} & 0 & 0 & 0 \\
\text { symm } & & & 44\left(1-D_{2}\right)\left(1-D_{3}\right) & 0 & 0 \\
& & & & 55\left(1-D_{1}\right)\left(1-D_{3}\right) & 0 \\
& & & & 66\left(1-D_{1}\right)\left(1-D_{2}\right)
\end{array}\right]
$$

where the assumption was made that the material principle directions coincide with the principle directions of damage. However, for a stiffness tensor defined in an arbitrary coordinate system, the damage effect tensor must be suitably transformed.

Thermodynamic force conjugate to the damage tensor $\mathbf{D}$ can be obtained if the Helmholtz and/or Gibbs free energies are defined in terms of stiffness and/or compliance tensors and substituted in the dissipation rate (3). Making use of the Legendre transformation:

$$
\begin{aligned}
\rho g\left(\boldsymbol{\sigma}, \mathbf{D}, \theta, \alpha^{\prime}, \boldsymbol{\alpha}^{\prime \prime}, \beta^{\prime}, \boldsymbol{\beta}^{\prime \prime}\right) & =\rho \psi\left(\boldsymbol{\varepsilon}_{e}, \mathbf{D}, \theta, \alpha^{\prime}, \boldsymbol{\alpha}^{\prime \prime}, \beta^{\prime}, \boldsymbol{\beta}^{\prime \prime}\right)-\boldsymbol{\sigma}: \boldsymbol{\varepsilon}_{e} \\
& =W\left(\boldsymbol{\varepsilon}^{e}, \theta, \mathbf{D}\right)+H_{p}\left(\theta, \alpha^{\prime}, \boldsymbol{\alpha}^{\prime \prime}\right)+H_{d}\left(\theta, \beta^{\prime}, \boldsymbol{\beta}^{\prime \prime}\right)-\boldsymbol{\sigma}: \boldsymbol{\varepsilon}_{e}
\end{aligned}
$$

and constitutive equation for stress and strain, one can define the Gibbs free energy in terms of damage, stress, temperature and the set of internal variables as: 


$$
\rho g\left(\boldsymbol{\sigma}, \mathbf{D}, \theta, \alpha^{\prime}, \boldsymbol{\alpha}^{\prime \prime}, \beta^{\prime}, \boldsymbol{\beta}^{\prime \prime}\right)=-\frac{1}{2} \boldsymbol{\sigma}:: \sigma \boldsymbol{\sigma}+H_{p}\left(\theta, \alpha^{\prime}, \boldsymbol{\alpha}^{\prime \prime}\right)+H_{d}\left(\theta, \beta^{\prime}, \boldsymbol{\beta}^{\prime \prime}\right)
$$

Rate of change of Gibbs energy is now obtained as:

$$
\begin{array}{r}
\rho g\left(\boldsymbol{\sigma}, \mathbf{D}, \theta, \alpha^{\prime}, \boldsymbol{\alpha}^{\prime \prime} \beta^{\prime}, \boldsymbol{\beta}^{\prime \prime}\right)=-\boldsymbol{\sigma}:: \quad: \boldsymbol{\sigma}-\frac{1}{2} \boldsymbol{\sigma}:: \boldsymbol{\sigma}+\rho \frac{\partial H_{p}}{\partial \alpha^{\prime}} \alpha^{\prime}+\rho \frac{\partial H_{p}}{\partial \boldsymbol{\alpha}^{\prime \prime}} \boldsymbol{\alpha}^{\prime \prime}+\rho \frac{\partial H_{d}}{\partial \beta^{\prime}} \beta^{\prime}+\rho \frac{\partial H_{d}}{\partial \boldsymbol{\beta}^{\prime \prime}} \boldsymbol{\beta}^{\prime \prime}+\rho \frac{\partial g}{\partial \theta} \theta \\
=-\boldsymbol{\sigma}:: \boldsymbol{\sigma}-\frac{1}{2} \boldsymbol{\sigma}: \quad(\mathbf{D}):: \frac{\partial \quad(\mathbf{D})}{\partial \mathbf{D}}: \boldsymbol{\sigma}: \mathbf{D}+\rho \frac{\partial H_{p}}{\partial \alpha^{\prime}} \alpha^{\prime}+\rho \frac{\partial H_{p}}{\partial \boldsymbol{\alpha}^{\prime \prime}} \boldsymbol{\alpha}^{\prime \prime}+\rho \frac{\partial H_{d}}{\partial \beta^{\prime}} \beta^{\prime}+\rho \frac{\partial H_{d}}{\partial \boldsymbol{\beta}^{\prime \prime}} \boldsymbol{\beta}^{\prime \prime}+\rho \frac{\partial g}{\partial \theta} \theta
\end{array}
$$

and further used in the expression for the adiabatic dissipation rate (5):

$$
\begin{aligned}
\Gamma= & \boldsymbol{\sigma}: \boldsymbol{\varepsilon}-\rho \psi-\rho \theta s=\boldsymbol{\sigma}: \boldsymbol{\varepsilon}-\left(\boldsymbol{\sigma}: \boldsymbol{\varepsilon}_{e}+\boldsymbol{\sigma}: \boldsymbol{\varepsilon}_{e}+\rho g\right)-\rho \theta s= \\
& =\left(\boldsymbol{\sigma}:-\boldsymbol{\varepsilon}_{e}\right): \boldsymbol{\sigma}-\rho\left(s+\frac{\partial g}{\partial \theta}\right) \theta+\boldsymbol{\sigma}: \boldsymbol{\varepsilon}_{p}-\rho \frac{\partial H_{p}}{\partial \alpha^{\prime}} \alpha^{\prime}-\rho \frac{\partial H_{p}}{\partial \boldsymbol{\alpha}^{\prime \prime}} \boldsymbol{\alpha}^{\prime \prime} \\
& +\frac{1}{2} \boldsymbol{\sigma}: \quad(\mathbf{D}):: \frac{\partial(\mathbf{D})}{\partial \mathbf{D}}: \boldsymbol{\sigma}: \mathbf{D}-\rho \frac{\partial H_{d}}{\partial \beta^{\prime}} \beta^{\prime}-\rho \frac{\partial H_{d}}{\partial \boldsymbol{\beta}^{\prime \prime}} \boldsymbol{\beta}^{\prime \prime} \geq 0
\end{aligned}
$$

The first two terms in the inequality above are constitutive equations for elastic strain and entropy (conjugate to (4)), hence the dissipation rate per unit initial volume is obtained as:

$$
\Gamma=\boldsymbol{\sigma}: \boldsymbol{\varepsilon}_{p}-\rho \frac{\partial H_{p}}{\partial \alpha^{\prime}} \alpha^{\prime}-\rho \frac{\partial H_{p}}{\partial \boldsymbol{\alpha}^{\prime \prime}} \boldsymbol{\alpha}^{\prime \prime}+\mathbf{Y}: \mathbf{D}-\rho \frac{\partial H_{d}}{\partial \beta^{\prime}} \beta^{\prime}-\rho \frac{\partial H_{d}}{\partial \boldsymbol{\beta}^{\prime \prime}} \boldsymbol{\beta}^{\prime \prime} \geq 0
$$

The force conjugate to damage tensor is hereinafter denoted as damage energy release rate and, from the expressions (5) and (23), can be written as:

$$
\mathbf{Y}=-\rho \frac{\partial \psi}{\partial \mathbf{D}}=\rho \frac{\partial g}{\partial \mathbf{D}}=\frac{1}{2} \boldsymbol{\sigma}: \quad(\mathbf{D}):: \frac{\partial \quad(\mathbf{D})}{\partial \mathbf{D}}: \boldsymbol{\sigma}
$$

A dissipative potential for damage is defined in terms of the damage energy release rate (24), as detailed in subsection 2.2.3.

\subsection{Orthotropic elastoplasticity with damage}

The thermodynamic state potentials, introduced in the subsection 2.1 , are defined for each irreversible process in terms of the two internal variables which describe isotropic and kinematic hardenings. However, the proposed model is limited to isotropic plastic and isotropic damage hardenings, respectively described with the scalar variables $\alpha=\alpha^{\prime}$ and 
$\beta=\beta^{\prime}$. The definitions of the dissipative potentials follow the constitutive equations which describe material elastic behaviour.

\subsubsection{Elastic behaviour}

As already stated, for damaged material the elastic strain energy is defined as $W\left(\boldsymbol{\varepsilon}, \boldsymbol{\varepsilon}_{p}, \mathbf{D}\right) \equiv \frac{1}{2}\left(\boldsymbol{\varepsilon}-\boldsymbol{\varepsilon}_{p}\right): \quad(\mathbf{D}):\left(\boldsymbol{\varepsilon}-\boldsymbol{\varepsilon}_{p}\right)=\frac{1}{2} \boldsymbol{\varepsilon}_{e}:(\mathbf{D}): \boldsymbol{\varepsilon}_{e} . \quad$ Consequently, the elastic constitutive relation depends on the degraded elastic stiffness:

$\boldsymbol{\sigma}=\frac{\partial W}{\partial \boldsymbol{\varepsilon}_{e}}=: \boldsymbol{\varepsilon}_{e}$

where $={ }^{-T}::^{-1}$ is defined by equation (15). The undamaged material stiffness elastic tensor is defined in terms of elastic material constants as:

$$
\left[\begin{array}{cccccc}
\frac{E_{1}\left(1-v_{32} v_{23}\right)}{\Delta_{c}} & \frac{E_{2}\left(v_{12}+v_{13} v_{32}\right)}{\Delta_{c}} & \frac{E_{3}\left(v_{13}+v_{12} v_{23}\right)}{\Delta_{c}} & 0 & 0 & 0 \\
\frac{E_{1}\left(v_{21}+v_{23} v_{31}\right)}{\Delta_{c}} & \frac{E_{2}\left(1-v_{13} v_{31}\right)}{\Delta_{c}} & \frac{E_{3}\left(v_{23}+v_{21} v_{13}\right)}{\Delta_{c}} & 0 & 0 & 0 \\
\frac{E_{1}\left(v_{31}+v_{21} v_{32}\right)}{\Delta_{c}} & \frac{E_{2}\left(v_{32}+v_{32} v_{23}\right)}{\Delta_{c}} & \frac{E_{3}\left(1-v_{21} v_{12}\right)}{\Delta_{c}} & 0 & 0 & 0 \\
0 & 0 & 0 & G_{23} & 0 & 0 \\
0 & 0 & 0 & 0 & G_{31} & 0 \\
0 & 0 & 0 & 0 & 0 & G_{12}
\end{array}\right]
$$

where:

$$
\Delta_{c}=1-v_{21} v_{12}-v_{31} v_{13}-v_{32} v_{23}-v_{12} v_{23} v_{31}-v_{21} v_{13} v_{32}
$$

The stiffness tensor is symmetric and positive definite, which impose some constrains on the material parameters [31].

\subsubsection{Plastic potential}

The evolution of the plastic deformation is controlled by a plastic potential introduced in the equation (8), through the associative flow law. The model, in its current form, allows only for the isotropic hardening. Consequently, the generalised plastic potential is a function of 
effective stress, thermodynamic force $R$ conjugate to hardening parameter $\alpha$ (introduced in (6) 1$)$, and temperature:

$F_{p}(\boldsymbol{\sigma}, R, \theta)=\bar{\sigma}_{F}-\left[\Delta R(\alpha, \theta)+R_{0}\right]=0$

where:

- $\bar{\sigma}_{F}$ is the effective equivalent plastic stress following Hill [37], which accounts for material orthotropy,

- $\quad R$ is thermodynamic hardening force defined by the MTS model [2].

The thermodynamic hardening force is decomposed onto the initial material hardening threshold (initial yield stress) $R_{0}$, and the increase of the yield stress due to hardening $\Delta R$, defined for a chosen reference direction. The referenced direction coincides with one of the material orthotropy principal directions (e.g. direction $a$ ).

The Hill's effective equivalent stress $\bar{\sigma}_{F}$ is defined as a function of deviatoric part of effective stress of damaged material as [37]:

$\bar{\sigma}_{F}=\left[\frac{1}{2} \boldsymbol{\sigma}^{\mathbf{T}}: \quad: \boldsymbol{\sigma}\right]^{1 / 2}=\left[\frac{1}{2} \boldsymbol{\sigma}^{\mathbf{T}}: \quad: \boldsymbol{\sigma}\right]^{1 / 2}$

where the effective plastic characteristic tensor is given by:

$$
={ }^{T}(\mathbf{D}): \quad: \quad(\mathbf{D})
$$

The positive definite tensor for orthotropic materials [37], is defined in the material principal coordinate system by:

$$
=\left[\begin{array}{cccccc}
G+H & -H & -G & 0 & 0 & 0 \\
-H & H+F & -F & 0 & 0 & 0 \\
-G & -F & F+G & 0 & 0 & 0 \\
0 & 0 & 0 & 2 N & 0 & 0 \\
0 & 0 & 0 & 0 & 2 L & 0 \\
0 & 0 & 0 & 0 & 0 & 2 M
\end{array}\right]
$$

where $F, G, H, L, M$ and $N$ are parameters characterising the current state of plastic anisotropy. The consistency between the general thermodynamic framework and treatment of 
anisotropic hardening requires tensor to be a state variable. However, the proposed model is intended for metals with a weak degree of anisotropy, which is not changed during the plastic deformation. Consequently, the assumption was made that that tensor is constant for the range of plastic deformations considered.

Instead of defining an explicit function for plastic hardening part of the free energy $H_{p}$, the derivative of which is the thermodynamic force $R$, the thermodynamic force is defined directly from the MTS model [2] as:

$R=\frac{\mu(\theta)}{\mu_{0}}\left[\sigma_{a}+\left[1-\left(\frac{k \theta \ln \left(\frac{\varepsilon_{0}}{\alpha}\right)}{\mu(\theta) b^{3} g_{0}}\right)^{\frac{1}{q}}\right]^{\frac{1}{p}} \sigma\right]$

where: $\sigma_{a}$ is athermal component of threshold stress, $\sigma$ is parameter of structure, $\varepsilon_{0}$ is reference strain rates and $\mu(\theta)$ temperature dependent shear modulus; the other parameters are material constants: $k$ is Boltzmann constant, $\mu_{0}$ is shear modulus at $0 K, b$ is Burgers vector, $g_{0}$ is normalized activation energy, $p$ and $q$ are the micromechanical constants. The athermal component of stress is assumed constant, and shear modulus in terms of temperature is calculated as proposed in [2]:

$$
\mu(\theta)=b_{0}-\frac{b_{1}}{\exp \left(\frac{b_{2}}{\theta}\right)-1}
$$

where $b_{0}, b_{1}$ and $b_{2}$ are material constants described below.

Having defined the plastic potential (28), the evolution equations for plastic strain rate and internal variable, $(10)_{1}$ and $(10)_{3}$, respectively, are defined from normality rule as:

$\boldsymbol{\varepsilon}_{p}=\lambda_{p} \frac{\partial F_{p}}{\partial \boldsymbol{\sigma}}=\frac{: \boldsymbol{\sigma}}{2 \bar{\sigma}_{F}} \lambda_{p}$ 
$\alpha=\lambda_{p} \frac{\partial F_{p}}{\partial R}=\lambda_{p}$

The plastic potential satisfies the Hertz - Signori - Moreau (Kuhn Tucker) conditions defined in (11). Lagrange multiplier is determined from the consistency condition which states that during the loading, the material state stays on the yield surface, i.e. $F_{p}(\sigma, R)=0$. Hence:

$\lambda_{p}=\frac{(D \mathbf{B}+C \mathbf{E}): \boldsymbol{\varepsilon}}{A D+C F}$

where

$A=\frac{\partial F_{p}}{\partial \boldsymbol{\sigma}}:: \frac{\partial F_{p}}{\partial \boldsymbol{\sigma}}-\frac{\partial F_{p}}{\partial R} \frac{\partial R}{\partial \alpha} \quad \mathbf{B}=\frac{\partial F_{p}}{\partial \boldsymbol{\sigma}}: \quad C=\frac{\partial F_{p}}{\partial \boldsymbol{\sigma}}: \frac{\partial}{\partial \mathbf{D}}: \frac{\partial F_{d}}{\partial \mathbf{Y}}: \boldsymbol{\varepsilon}_{e}$

$D=\boldsymbol{\sigma}: \quad(\mathbf{D}) \frac{\partial F_{d}}{\partial \mathbf{Y}}:: \frac{\partial(\mathbf{D})}{\partial \mathbf{D}}: \boldsymbol{\sigma}+\boldsymbol{\sigma}: \quad(\mathbf{D})::\left(\frac{\partial^{2}(\mathbf{D})}{\partial \mathbf{D} \partial \mathbf{D}} \frac{\partial F_{d}}{\partial \mathbf{Y}}: \boldsymbol{\sigma}+\frac{\partial(\mathbf{D})}{\partial \mathbf{D}}: \frac{\partial}{\partial \mathbf{D}}: \frac{\partial F_{d}}{\partial \mathbf{Y}}\right)+\frac{\partial F_{d}}{\partial B} \frac{\partial B}{\partial \beta}$

$\mathbf{E}=\boldsymbol{\sigma}: \quad(\mathbf{D}):: \frac{\partial(\mathbf{D})}{\partial \mathbf{D}}: \quad F=\boldsymbol{\sigma}: \quad(\mathbf{D}):: \frac{\partial(\mathbf{D})}{\partial \mathbf{D}}:: \frac{\partial F_{p}}{\partial \boldsymbol{\sigma}}$

The update of the yield surface radius in the proposed model is based on the evolution equation for the threshold stress of the MTS model, i.e. the hardening modulus calculated for the reference direction, which represents the slope of the inelastic portion of a uniaxial stress vs. plastic strain curve:

$E_{T, i}^{a}=\frac{\mu}{\mu_{0}} \Theta_{0}\left(1-\frac{\tanh \left(\delta \frac{\sigma-\sigma_{a}}{\sigma_{s}-\sigma_{a}}\right)}{\tanh (\delta)}\right]\left[1-\left(\frac{k \theta \ln \left(\frac{\varepsilon_{0}}{\alpha}\right)}{\mu(\theta) b^{3} g_{0}}\right)^{\frac{1}{q}}\right]^{\frac{1}{p}}$

where $\sigma_{s}$ is saturation threshold stress and $\delta$ is a fitting constant. Finally, the evolution of the yield surface, i.e. $\Delta R$, for the reference direction is determined as:

$R_{a}=R_{0}+\sum_{i=1}^{n} \frac{E_{a} E_{T, i}^{a}}{E_{a}-E_{T, i}^{a}} \Delta \varepsilon_{i}=R_{0}+\sum_{i=1}^{n} E_{p, i}^{a} \Delta \varepsilon_{i}$

where: $E_{p, i}^{a}=\frac{E_{a} E_{T, i}^{a}}{E_{a}-E_{T, i}^{a}}$ and $E_{a}$ are the tangent and elastic modulus in the $a$ direction, respectively. The equation (38) completes the description of the plastic model. 


\subsubsection{Damage potential}

The evolution of damage is described by an isotropic damage hardening variable $\beta$, which is derived from the modified Klepaczko model [1]. As with the plastic dissipative potential, it can be assumed that there exists a surface $F_{d}(\mathbf{Y}, B, \theta)=0$, which separates the damaged from the undamaged state of the material [17]. A damage criterion is a quadratic homogeneous function of the damage energy release rate, given in the following general form:

$$
F_{d}(\mathbf{Y}, B, \theta)=Y_{e q}-\left[B(\beta, \theta)+B_{0}\right]
$$

Where $Y_{e q}$ is the equivalent damage energy release rate, $B_{0}$ initial damage threshold, and $B(\beta, \theta)$ is thermodynamic force conjugate to damage variable that describes the size of the damage surface. The equivalent damage energy release rate $Y_{e q}$ is defined by:

$$
Y_{e q}=\left[\frac{1}{2} \mathbf{Y}^{\mathbf{T}}: \quad: \mathbf{Y}\right]^{1 / 2}
$$

where is damage characteristic tensor, which is a symmetric tensor of the same order as the plastic characteristic tensor and allows for the modelling of anisotropic damage.

The characteristic tensor is determined from the experimental data where the material principle directions of orthotropy coincide with the principle direction of damage (see subsection 2.2.4). Consequently, $Y_{12}=Y_{23}=Y_{13}=0$ and the damage characteristic tensor reduces to the second order tensorial form, originally proposed by Zhu and Cescotto [17]:

$$
\mathbf{J}=2\left[\begin{array}{ccc}
J_{1} & \sqrt{J_{2}} & \sqrt{J_{3}} \\
\sqrt{J_{2}} & J_{2} & \sqrt{J_{2} J_{3}} \\
\sqrt{J_{3}} & \sqrt{J_{2} J_{3}} & J_{3}
\end{array}\right]
$$

where: $J_{i}, i=1,2,3$ are material parameters.

The internal variable for damage hardening is derived starting from the commonly used failure criteria which assume that duration of loading (time) and the load magnitude (stress) are key parameters in material dynamic failure. A general method proposed by Tuler and 
Butcher [38] and by Gilman and Tuler [39], suggests the use of a damage function $\phi$, as a function of the entire loading (stress) history $\sigma(t)$ :

$\phi=\int_{0}^{t} f[\sigma(t)] d t$

where $\phi$ is selected to be any convenient function of damage and $t$ is time. When $\phi$ reaches a critical value, $t$ becomes the time to failure $t_{c}$. For example, $\phi$ could be total number or volume of microcracks in the material at failure.

Tuler and Butcher suggested a general criterion based on the concept of cumulative damage and they found that function $\phi$ can be explained in powers of $\sigma-\sigma_{0}$, where $\sigma_{0}$ is the threshold stress below which no damage occurs, regardless the stress duration [39]. It was further suggested that one term might be expected to be dominant. The authors proposed a relationship between various loading conditions and spall fracture, including time dependence of spalling in the form of generalised criterion, where the spall stress depends on the stress pulse duration:

$\int_{0}^{t_{c}}\left(\sigma(t)-\sigma_{0}\right)^{\lambda} d t=\phi$

where $\lambda$ and $\phi$ are material constants determined experimentally, $\sigma_{0}$ is threshold stress and $t_{c}$ is the time to failure.

To account for the physical damage mechanisms associated with the material separation during fracture processes, the concept of thermal activation was introduced by Zhurkov, [40]. It is generally accepted that rate and temperature effects are related to thermally activated micromechanical processes of plasticity and damage. The creation of free surfaces in a stressed body occurs with assistance of thermally activated processes, or in other words, thermal vibration of the crystalline lattice provides some additional energy to promote 
fracture [41]. The kinetic concept of the mechanism of fracture is understood to be a time dependant process, for which the rate is determined by stress and temperature.

Zhurkov proposed the following kinetic equation which gives the relationship between the lifetime $t_{c}$, the critical stress $\sigma$ and absolute temperature $\theta$ [40]:

$t_{c}=t_{c o} \exp \left(\frac{U-\gamma \sigma}{k \theta}\right)$

where $k$ is Boltzmann's constant, $t_{c 0}$ is the period of vibration of atoms in solids, $U_{0}$ can be interpreted as the magnitude of the energy barrier related to the probability of bond breaking, and $\gamma$ is the activation volume linked to the microstructure. In this expression, $t_{c 0}, U_{0}$ and $\gamma$ represent material parameters. The dependence of the effective barrier energy $\Delta U=U_{0}-\gamma \sigma$ on the stress, accounts for acceleration of the fracture process in a stressed body, and a decrease in the time to failure for highly stressed materials.

The form (44) can be obtained by considering bond-breaking and assuming that this process is a fundamental mechanism of fracture initiation. In this approach, fractures form and propagate because the rate of bond breaking is greater than the rate of bond healing. Tobolsky and Eyring originally developed this theory for polymeric threads [42]. However, it has been shown by other investigators that this theory is equally valid for crystalline and amorphous materials.

At large constant stresses which acts on $N$ bonds per unit area and low temperatures, when the bond healing process does not take place, an expression for net bond breaking can be written as:

$-\frac{d N}{d t} \approx N \frac{k \theta}{h} \exp \left(-\frac{U}{k \theta}\right) \exp \left(\frac{\lambda \sigma}{N k \theta}\right)$

where: $h$ is Planck's constant and $U$ represents the activation free energy.

After integration of the above equation, the lifetime $t_{c}$ of a specimen subject to a constant stress can be expressed as: 


$$
\ln t_{c}=\ln \frac{h N_{0}}{\lambda \sigma}+\frac{U}{k \theta}-\frac{\lambda \sigma}{N_{0} k \theta}
$$

which can be rearranged in the form that was used by Zhurkov, in terms of the time to failure:

$$
t_{c}=\frac{h N_{0}}{\lambda \sigma} \exp \left(\frac{U-\lambda \sigma / N_{0}}{k \theta}\right)=t_{c o} \exp \left(\frac{U-\gamma \sigma}{k \theta}\right)
$$

Dremin and Molodets proposed a modification to the Zhurkov criterion for applications requiring modelling of spall failure [43]. The proposed modification takes into account the local effects due to the barriers being overcome homogeneously in localised micro-volumes during the fracture process. This means that the activation energy has to be a function of the local stress and the local activation volume. The authors also used the fact that in ductile materials that undergo significant plastic deformation before failure, failure does not occur instantaneously, but rather after a certain cumulative time. This provided a basis for the introduction of the damage accumulation rate function $\phi$, which characterises the thermallyactivated stage of fracture:

$$
\phi=\phi_{0} \exp \left(-\frac{\Delta U}{k \theta}\right) \quad \text { and } 1=\int_{0}^{t_{f}} \phi d t
$$

where an assumption was made that variables $\dot{\phi}_{0}=1 / t_{0}, U_{0}, \quad \beta$ do not depend on temperature, and $\Delta U(\sigma)$ is the stress dependent free energy of activation. It was also assumed that the time interval of micro crack growth is very short (typically a few nanoseconds in hard materials), in comparison with the critical time to spalling.

Klepaczko, following Zhurkov's work, proposed a cumulative criterion for short and very short loading times, where thermally activated rate processes are also the physical basis for this model [44]. It has been demonstrated that the model can accurately predict the critical time $t_{c}$, as a function of the spall (tensile) stress $\sigma$ for different materials [44]. 
By substituting Yokobori's expression for the activation energy $\Delta U(\sigma)=\Delta U_{0} \ln \left(\sigma_{0} / \sigma\right)$ into Dremin and Molodets' cumulative damage criterion, and introducing a factor $\alpha\left(\Delta U_{0}, \theta\right)$ that depends upon the activation energy $\Delta U_{0}$ for a stress free body and temperature, Klepaczko developed the following failure criterion:

$\int_{0}^{t_{c}}\left(\frac{\sigma(t)}{\sigma_{0}}\right)^{\alpha\left(\Delta U_{0}, \theta\right)} d t=t_{c 0} \quad t_{c} \leq t_{c 0} \quad \sigma \geq \sigma_{0}$

where $\sigma_{0}$ is the threshold stress corresponding to the characteristic time $t_{c 0}, t_{c 0}$ is the longest critical time when $\sigma\left(t_{c 0}\right)=\sigma_{0}$, for $t_{c} \geq t_{c 0}, \sigma=\sigma_{0}$ and $\sigma_{0}=$ const, $\alpha\left(\Delta U_{0}, \theta\right)=\frac{\Delta U_{0}}{k \theta}$. The material parameters $\sigma_{0}, t_{c 0}, \alpha\left(\Delta U_{0}, \theta\right)$ are measured at constant temperature $\theta$.

When the deformation process are non-isothermal, e.g. spall failure, the exponent $\alpha\left(\Delta U_{0}, \theta\right)$ becomes time dependent, through changes of temperature during loading $\alpha\left(\Delta U_{0}, \theta(t)\right)$. In such a case, the proposed cumulative criterion must be integrated accordingly, including the temperature time dependency $\theta(t)$.

This failure criterion includes both the brittle mechanisms of micro-cracking and material separation and dynamic plasticity. These two processes are combined in different proportions determined by the value of exponent $\alpha$. An increase in temperature results in an increase of the threshold failure stress, due to increased levels of material ductility.

Further development of this criterion was done by Hanim and Klepaczko [45], through the introduction of a temperature dependant threshold stress $\sigma_{0}$ :

$\sigma_{0}(\theta)=\sigma_{0}^{0} \frac{\mu(\theta)}{\mu_{0}}$

where $\sigma_{0}^{0}$ is the threshold stress at absolute temperature near $0 K, \mu(\theta)$ is temperature dependant shear modulus, and $\mu_{0}$ is shear modulus near $0 K$. 
Following the work of Kocks [2], the normalised activation energy $u_{0}$, can be defined as:

$u_{0}=\frac{\Delta U_{0}}{\mu(\theta) b^{3}}$

where $b$ is burgers vector. In choosing this definition of normalised damage activation energy, the damage model is made consistent with the MTS model. Using this type of normalisation, one can define the stress dependent activation energy given by Yokobori in the following form:

$$
\Delta U(\sigma)=\mu(\theta) b^{3} u_{0} \ln \left(\frac{\sigma_{0}^{0} / \mu_{0}}{\sigma / \mu(\theta)}\right)
$$

Substitution of the expression for the activation energy (51) into the modified Klepaczko's failure criterion given by equation (49), yields:

$\int_{0}^{t_{c}}\left\langle\left.\frac{d \sigma}{d \varepsilon}\right|_{C}-\frac{d \sigma}{d \varepsilon}\right\rangle\left(\frac{\sigma(t) / \mu(\theta)}{\sigma_{0} / \mu_{0}}\right)^{\left(\frac{\mu(\theta) b^{3} u_{0}}{k \theta}\right)} d t=t_{c 0}$

where: the Macaulay brackets $\langle x\rangle$ denotes Heaviside function equal to 1 for $x \geq 0$ and 0 for $x<0 ; t_{c 0}, \sigma_{0}$ and $u_{0}$ have to be determined from experimental data, $\sigma_{0}=\sigma_{0}\left(\left.\frac{d \sigma}{d \varepsilon}\right|_{C}\right)$ is the damage threshold stress, determined by the critical plastic hardening rate, $\left.\frac{d \sigma}{d \varepsilon}\right|_{C}$ (set to 0.01 for the simulations presented in this paper); and $t_{c 0}$ is the maximum time to failure. Finally, the internal variable for the isotropic damage hardening is defined as:

$$
\beta=\frac{\int_{0}^{t_{c}}\left\langle\left.\frac{d \sigma}{d \varepsilon}\right|_{C}-\frac{d \sigma}{d \varepsilon}\right\rangle\left(\frac{\sigma(t) / \mu(\theta)}{\sigma_{0} / \mu_{0}}\right)^{\left(\frac{\mu(\theta) b^{3} u_{0}}{k \theta}\right)} d t}{t_{c 0}}
$$

Derivation of the equation (54) is given in the Appendix A at the end of this paper. 
Thermodynamic force $B$, is assumed to be a linear function of the internal variable for isotropic damage hardening (54). This constitutive equation is given at the end of the following subsection, where the material characterisation of the damage model is illustrated.

The evolution of anisotropic damage is characterised by the damage flow rule:

$\mathbf{D}=-\lambda_{d} \frac{\partial F_{d}}{\partial \mathbf{Y}}=-\frac{\mathbf{J}: \mathbf{Y}}{2 Y_{e q}} \lambda_{d}$

and damage hardening rule:

$\beta=-\lambda_{d} \frac{\partial F_{d}}{\partial B}=\lambda_{d}$

Damage loading/unloading rule:

$F_{d} \leq 0 \quad \dot{\lambda}_{d} \geq 0 \quad \dot{\lambda}_{d} F_{d}=0$

Similarly to plasticity, the Lagrange multiplier for evolution of damage is determined from the consistency condition, i.e. $F_{d}=0$, which leads to the following general expression:

$\lambda_{d}=\frac{(A \mathbf{E}-F \mathbf{B}): \boldsymbol{\varepsilon}}{A D+C F}$

where:

$A=\frac{\partial F_{p}}{\partial \boldsymbol{\sigma}}:: \frac{\partial F_{p}}{\partial \boldsymbol{\sigma}}-\frac{\partial F_{p}}{\partial R} \frac{\partial R}{\partial \alpha} \quad \mathbf{B}=\frac{\partial F_{p}}{\partial \boldsymbol{\sigma}}: \quad C=\frac{\partial F_{p}}{\partial \boldsymbol{\sigma}}: \frac{\partial}{\partial \mathbf{D}}: \frac{\partial F_{d}}{\partial \mathbf{Y}}: \boldsymbol{\varepsilon}_{e}$

$D=\boldsymbol{\sigma}: \quad(\mathbf{D}) \frac{\partial F_{d}}{\partial \mathbf{Y}}:: \frac{\partial(\mathbf{D})}{\partial \mathbf{D}}: \boldsymbol{\sigma}+\boldsymbol{\sigma}: \quad(\mathbf{D})::\left(\frac{\partial^{2}(\mathbf{D})}{\partial \mathbf{D} \partial \mathbf{D}} \frac{\partial F_{d}}{\partial \mathbf{Y}}: \boldsymbol{\sigma}+\frac{\partial(\mathbf{D})}{\partial \mathbf{D}}: \frac{\partial}{\partial \mathbf{D}}: \frac{\partial F_{d}}{\partial \mathbf{Y}}\right)+\frac{\partial F_{d}}{\partial B} \frac{\partial B}{\partial \beta}$

$\mathbf{E}=\boldsymbol{\sigma}: \quad(\mathbf{D}):: \frac{\partial(\mathbf{D})}{\partial \mathbf{D}}: \quad F=\boldsymbol{\sigma}: \quad(\mathbf{D}):: \frac{\partial(\mathbf{D})}{\partial \mathbf{D}}:: \frac{\partial F_{p}}{\partial \boldsymbol{\sigma}}$

The last equation completes the description of the damage part of the proposed model. The following subsection describes the material characterisation of the damage potential.

\subsubsection{Experimental characterisation of the material model parameters}

\section{Calibration of the MTS model parameters}

As described in the Introduction, the calibration of the constitutive model was carried out for a range of strain rates, which are combined with different temperature levels, in order to 
represent evolution of plastic deformation (in other words flow stress) equivalent to the high strain rate loading. Consequently, the MTS part of the constitutive model was calibrated for a range of strain rates between $6.4 \times 10^{-4} s^{-1}$ and $6.4 \times 10^{1} s^{-1}$ and range of temperatures from $223.15 K$ to $473.15 K$. Few MTS approximations and their corresponding experimental curves are shown in Figure 1. As it can be seen in the figure, the same material parameters provided a good approximation of the tensile test performed at $6.4 \times 10^{2} \mathrm{~s}^{-1}$ and initial temperature $\theta=288.15 \mathrm{~K}$. This was the first indication that the material parameters can be used to simulate behaviour of the material at the high rates of loading.

\section{Near Figure 1}

In order to further validate the basic assumption used in the MTS model calibration that the effect of lowering the test temperature are similar to increase in the test loading rate the flow stress - plastic strain curves obtained at room temperature and high loading rate were compared with the corresponding curves obtained at lower temperatures and lower strain rates. For instance the flow stress - plastic strain curves for the loading rate $\varepsilon=6.4 \times 10^{3} \mathrm{~s}^{-1}$ at $\theta=293.15 \mathrm{~K}$ and the loading rate $\varepsilon=64 \mathrm{~s}^{-1}$ at $\theta=223.15 \mathrm{~K}$ are given in Figure 2.

\section{Near Figure 2}

The good agreement between the high strain rate and low strain rate curves with the maximum difference below 4\% (as shown in the Figure 2) indicates that the material model parameters, derived as described above, can be used in the simulation of the Taylor anvil impact tests. The strain rates recorded in our simulation of the test stay below $6.0 \times 10^{3} \mathrm{~s}^{-1}$ as it can be seen in Appendix B. 


\section{Characterisation of the material damage parameters}

The material parameters that constitute the damage characteristic tensor are obtained from the equivalence of work done by damage energy release rate. The change in the equivalent damage energy release rate in any principal direction of material anisotropy depends upon the total amount of damage work done by the corresponding damage component. For an equivalent variation, the damage work done by each component should be the same [17]. In the case of a piecewise linear damage hardening shown in Figure 3, the damage work in component 1 is:

$W_{d 1}=\frac{1}{2} D_{1}\left(Y_{10}+Y_{1}\right)=\frac{1}{2} \frac{\left(Y_{1}-Y_{10}\right)}{D_{t 1}}\left(Y_{10}+Y_{1}\right)=\frac{1}{2 D_{t 1}}\left(Y_{1}^{2}-Y_{10}^{2}\right)$

Similarly, the damage work done in terms of equivalent damage energy release rate $Y_{e q}$ is given by:

$W_{d e q}=\frac{1}{2 D_{t}}\left(Y_{e q}^{2}-Y_{0}^{2}\right)$

Near Figure 3

Equating (60) and (61) reveals,

$J_{1}=\left(\frac{Y_{e q}}{Y_{1}}\right)^{2}=\frac{Y_{e q}^{2}}{\left(D_{t 1} / D_{t}\right)\left(Y_{e q}^{2}-Y_{0}^{2}\right)+Y_{10}^{2}}$

Similarly, one can obtain:

$$
\begin{aligned}
& J_{2}=\left(\frac{Y_{e q}}{Y_{2}}\right)^{2}=\frac{Y_{e q}^{2}}{\left(D_{t 2} / D_{t}\right)\left(Y_{e q}^{2}-Y_{0}^{2}\right)+Y_{20}^{2}} \\
& J_{3}=\left(\frac{Y_{e q}}{Y_{3}}\right)^{2}=\frac{Y_{e q}^{2}}{\left(D_{t 3} / D_{t}\right)\left(Y_{e q}^{2}-Y_{0}^{2}\right)+Y_{30}^{2}}
\end{aligned}
$$

If component 1 coincides with the reference direction, then

$$
\begin{aligned}
& J_{1}=1 \\
& J_{2}=\frac{Y_{1}^{2}}{\left(D_{t 2} / D_{t 1}\right)\left(Y_{1}^{2}-Y_{10}^{2}\right)+Y_{20}^{2}}
\end{aligned}
$$




$$
J_{3}=\frac{Y_{1}^{2}}{\left(D_{t 3} / D_{t 1}\right)\left(Y_{1}^{2}-Y_{10}^{2}\right)+Y_{30}^{2}}
$$

In the above equations, the damage hardening rates $D_{t i}$ are the slopes of $Y_{i}-D_{i}$ curves for the $i$ direction, where $Y_{i}$ is the current damage energy density release rate corresponding to the $i$ direction and $Y_{i 0}$ is the initial damage energy density release rate corresponding to the $i$ direction.

In this work, the damage characteristic tensor $\mathbf{J}$ was assumed constant (the same assumption was made for the plastic characteristic tensor ). This assumption significantly simplifies the model and the process of material experimental characterisation. In spite of this simplification, the comparisons between simulation results and experimental data presented in the model validation section shows good agreement. To determine components of the $\mathbf{J}$ tensor for a specific material, experimental damage energy density release rate verses damage $\left(Y_{i}\right.$ vs. $\left.D_{i}\right)$ curves are required.

Using the definition of damage energy density release rate (24), its principle components can be expressed as:

$$
-Y_{i}=\frac{\sigma_{i}}{1-D_{i}} \frac{C_{i j}^{-1}}{\left(1-D_{i}\right)\left(1-D_{j}\right)} \sigma_{j}
$$

where $i=1,2,3$ and the stress and material stiffness tensors are represented in the Voigt notation. When applied to the uniaxial stress state (tensile test data) in a direction $i$, the equation (66) reduces to (no summation with respect to $i$ ):

$$
-Y_{i}\left(D_{i}\right)=\frac{\sigma_{i i}^{2}(\varepsilon)}{E_{i}\left(1-D_{i}(\varepsilon)\right)^{3}}
$$

The $D_{i}(\varepsilon)$ relationships can be derived from (54), by using the relationships for the strain rates, i.e. $t=\varepsilon / \varepsilon, t_{c}=\varepsilon_{c} / \varepsilon$, which result in: 
$D_{i}\left(\varepsilon_{i i}\right)=\frac{\left[\int_{0}^{\varepsilon}\left\langle\left.\frac{d \sigma}{d \varepsilon}\right|_{C}-\frac{d \sigma}{d \varepsilon}\right)\left(\frac{\sigma_{i}(\varepsilon) / \mu(\theta)}{\sigma_{0} / \mu_{0}}\right)^{\left(\frac{\mu(\theta) b^{3} u_{0}}{k \theta}\right)} d \varepsilon\right]}{\varepsilon_{c 0}}$

The relationships (67) and (68) enables the characterisation of the damage model from the uniaxial stress state (tensile test data). By integrating the equation (68), one obtains the expression, convenient when interpreting tensile test data:

$D_{i}\left(\varepsilon_{i i}\right)=\frac{\varepsilon}{\varepsilon_{c 0}} \frac{1}{\left(\left(\frac{\mu(\theta) b^{3} u_{0}}{k \theta}\right)+1\right)}\left(\frac{\sigma_{i}(\varepsilon) / \mu(\theta)}{\sigma_{0} / \mu_{0}}\right)^{\left(\frac{\mu(\theta) b^{3} u_{0}}{k \theta}\right)}$

Using experimental data from uniaxial tensile tests $\left(\sigma_{i i}\left(\varepsilon_{i i}\right)\right.$ curves) for AA7010, the $D_{i}\left(\varepsilon_{i i}\right)$ curves, shown in Figure 4 and Figure 5, were calculated using equation (69).

Near Figure 4

Near Figure 5

From the damage-strain curves it can be seen that $D_{1}$ grows more rapidly then $D_{2}$, i.e. $\dot{D}_{1}>\dot{D}_{2}$. At the point of fracture, $D_{1}=0.108$ and $D_{2}=0.141$.

Knowing $D_{i}\left(\varepsilon_{i i}\right)$ and $\sigma_{i i}\left(\varepsilon_{i i}\right)$ relationships and combining with equation (67), which defines the relationship between the damage energy release rate and damage, $Y_{i}\left(D_{i}\right)$ curves for $i=1$ and $i=2$ calculated. For instance, the $Y_{i}\left(D_{i}\right)$ curves for $\varepsilon=6.4 s^{-1}$ and range of temperatures from $223.15 K$ to $343.15 K$ are shown in Figure 6 and Figure 7. 
Near Figure 6

Near Figure 7

The proposed damage model and the procedure for calibration of the model were applied to aluminium alloy AA7010. The ability of the model to reproduce experimental data from the tensile tests is illustrated by the stress strain curves for strain rate $\varepsilon=6.4 \mathrm{~s}^{-1}$ and temperatures 223.15 $K$ and $343.15 K$ shown in Figure 8 and Figure 9, respectively.

One can observe that the simulation results agree well with the experimental data, due to the observed decrease in stress (material softening) caused by damage growth within the material. In addition, effective stress-strain curves using the pure implementation of the MTS with no damage included, demonstrates clearly the limitations with this model as no material softening can occur.

Near Figure 8

Near Figure 9

Using the plasticity model and the model calibration procedures presented, $\sigma_{22} \varepsilon_{22}$ stressstrain curves shown in Figure 9 and Figure 11, were calculated for different temperature and strain rate levels.

Near Figure 10 
Near Figure 11

Table 1 and Table 2 provide a complete set of model parameters derived for AA7010. As no information on $\left(\sigma_{33} \varepsilon_{33}\right)$ curves were available, parameters in the principal direction 3 were assumed to be equal to the parameters in the reference direction.

Table 1 Material model parameters - Elastic moduli

\begin{tabular}{lll}
\hline Parameter & Description & Nominal value \\
\hline$E_{1}$ & Initial elastic modulus & $71,100 \mathrm{GPa}$ \\
$E_{2}$ & Initial elastic modulus & $70,326 \mathrm{GPa}$ \\
$E_{3}$ & Initial elastic modulus & $71,100 \mathrm{GPa}$ \\
\hline
\end{tabular}

Table 2 Damage parameters

\begin{tabular}{lll}
\hline Parameter & Description & Nominal value \\
\hline$Y_{10}$ & Initial damage energy density release rate & $8.62 \mathrm{MPa}$ (at 0 K) \\
$D_{t 1}$ & Damage hardening & $10.29 \mathrm{MPa}$ \\
$Y_{20}$ & Initial damage energy density release rate & $8.78 \mathrm{MPa}$ (at 0 K) \\
$D_{t 2}$ & Damage hardening & $5.82 \mathrm{MPa}$ \\
$Y_{30}$ & Initial damage energy density release rate & $8.62 \mathrm{MPa}$ (at 0 K) \\
$D_{t 3}$ & Damage hardening & $10.29 \mathrm{MPa}$ \\
\hline
\end{tabular}

\section{Model Validation - Taylor Anvil Test}

\subsection{Experimental procedure and results}

Taylor cylinder specimens were manufactured from AA7010 rolled plate, with a diameter $D=9.30 \mathrm{~mm}$ and length $L=46.50 \mathrm{~mm}$, giving a length-to-diameter ratio, $L / D=5$. The experimental coordinate system $(\mathrm{X}, \mathrm{Y}, \mathrm{Z})$ adopted for the test had the $\mathrm{Z}$ axis aligned with the longitudinal cylinder direction, as shown in Figure 12.

Near Figure 12 
A number of Taylor tests were performed at velocity of $200 \mathrm{~m} / \mathrm{s}$, using a smooth-bore, single-stage gas gun. Figure 13 provides photographs of the side profile and footprint of the recovered sample. Final specimen height was $42.2 \mathrm{~mm}$. The observed footprint had an elliptical shape, which was a direct consequence of material orthotropy.

Near Figure 13

\subsubsection{Experimental test results}

A post-test deformed specimen was scanned by using a 3D scanning machine, which enabled a digitised side profile and footprint cross-sectional area at the impact interface to be generated. Eccentricity, defined as a ratio of major to minor diameters, for the specimen impacted at $200 \mathrm{~m} / \mathrm{s}$ is 1.04 . A comparison of minor and major side profiles of post-test geometry plotted as radial strain vs. distance is illustrated in Figure 15.

Near Figure 14

Near Figure 15

\subsection{Taylor anvil test simulation}

The new material model was implemented in DYNA3D nonlinear hydrocode, available from Lawrence Livermore National Laboratory [8], where the integration of the state variables is done explicitly by using the central difference method.

Stress update is performed using, for this purpose developed, elastic predictor/plastic-damage corrector mapping integration algorithm. Stress update begins with the calculation of the (trial) stress defined by equation (25), assuming that the total strain increment is elastic. This trial stress is further used for the calculation of the plastic and damage potentials (28) and (39) 
, respectively. If the material state is inside of the both plastic and damage surface, the deformation process is elastic, and consequently the trial stress becomes a stress at the end of the time increment. However, if the material state is outside of the yield surface, the Lagrange multiplier (35), plastic strain tensor (34) and yield surface (38) are calculated so that the consistency condition are satisfied and the material state is returned to the yield surface. The criterion for damage initiation is defined in terms of critical plastic hardening rate, whereas the damage evolution is determined by the Lagrange multiplier (58) and the consistency condition of the damage process. The algorithm for the stress update is coupled with the EOS and algorithm for the integration of momentum equation.

The model was validated by numerical simulations of Taylor anvil tests for AA7010, with the specimen geometry described in the previous subsection. The macroscopic strain rate for the impact velocity $200 \mathrm{~m} / \mathrm{s}$ was $4.3 \times 10^{3} \mathrm{~s}^{-1}$; the calculated local strain rate histories for a number of locations in the cylinder are given in the Appendix B.

In the simulations, the $c m-g-\mu s$ system of units was used. The proposed material model was used in conjunction with the Gruneisen Equation of State, where the material parameters can be found in Table 3.

Table 3 Grunisen EOS constants

\begin{tabular}{lll}
\hline Parameter & Description & Nominal value \\
\hline $\mathrm{C}_{0}$ & Bulk sound speed & $0.5386 \mathrm{~cm} / \mathrm{s}$ \\
$\mathrm{S}_{1}$ & First Hugoniot slope coefficient & 1.339 \\
$\mathrm{~S}_{2}$ & Second Hugoniot slope coefficient & 0 \\
$\mathrm{~S}_{30}$ & Third Hugoniot slope coefficient & 0 \\
$\gamma_{0}$ & Gruneisen coefficient & 1.97 \\
$\mathrm{~B}$ & First order volume correction coefficient & 0.48 \\
$\mathrm{E}_{0}$ & Initial internal energy & 0.0 \\
$\mathrm{~V}_{0}$ & Initial relative volume & 1.0 \\
\hline
\end{tabular}


On the basis of published data for the spall strength of aluminium alloy AA 7010 [46], parameters which are included in the proposed failure criterion were determined:

- Threshold stress $\sigma_{0}=1.05 G P a$

- $\quad$ Normalised activation energy $u_{0}=0.0087$

- Critical time $t_{c 0}=2 \mu s$

Values for the other parameters include:

- Boltzmann's constant $k=1.38 \times 10^{-23} \mathrm{~J} / \mathrm{K}$,

- Burger's vector $b=0.286 \times 10^{-9} \mathrm{~m}$

- Constants for the shear modulus function: $b_{0}=28.83 \mathrm{GPa}$ is shear modulus at $0 \mathrm{~K}$, $b_{1}=4.45 G P a$ and $b_{2}=248.5 K$.

In order to reduce the number of elements and the associated CPU cost of the simulations, a quarter of the Taylor cylinder was generated using a uniform solid butterfly mesh.

Near Figure 16

The post-test distribution of damage in Taylor specimen, for the impact velocity of $200 \mathrm{~m} / \mathrm{s}$ is shown in Figure 16. Once the damage initiation criterion is reached, the model calculates the evolution of damage and when the failure criterion is satisfied, the deviatoric stresses are set to zero, resulting in the failed material only resisting compressive loading. When an element fails due to accumulated damage, the element is removed from calculation.

The simulation results and the test data for the specimen post-test profile given in Figure 17 illustrate good agreement. It can be concluded that the proposed model is capable of capturing more accurately major and minor distributions of plastic strains, and furthermore, the proposed model can describe the evolution of damage adequately. 


\section{Summary and conclusions}

An anisotropic thermoelastic-plastic material model with damage was developed within the general framework of continuum thermodynamics for irreversible processes, by defining a set of internal state variables and their conjugate generalised forces.

The proposed framework is capable of accommodating:

- General nonlinear elastic-plastic response

- Coupling of damage and plasticity,

- Temperature and strain rate dependent isotropic strain hardening, based on the MTS model

- Linear damage hardening with temperature dependant initial damage threshold. The damage evolution law was developed by adopting the damage surface concept and Klepaczko's cumulative damage theory [44].

Material anisotropy is considered for elastic, plastic and damage responses. In the elastic regime, material anisotropy is modelled through the appropriate material elastic stiffness (compliance) tensor . Anisotropic plasticity is considered through Hill's yield function, i.e. the plastic characteristic tensor , and in the damage regime, through a damage characteristic tensor $\mathbf{J}$ in the damage evolution law. The damage characteristic tensor $\mathbf{J}$ was determined using the principle of equivalence of damage work.

The new material model was implemented in DYNA3D, using for this purpose, a developed elastic predictor/plastic corrector/damage mapping integration algorithm. The material model parameters were obtained from the series of tensile tests conducted for a range of strain rates and a range of temperatures. The model was validated by numerical simulations of a tensile test at a strain rate $6.4 \times 10^{2} s^{-1}$ and a Taylor anvil test for AA7010.

Good agreement for the predicted post-test profile was achieved, and it can be concluded that the proposed model can capture more accurately, major and minor distributions of plastic strains, and furthermore, the proposed model can describe the evolution of damage adequately. 


\section{References}

[1] Klepaczko, J. R. (1990), "Dynamic crack initiation, some experimental methods and modelling", in Klepaczko, J. R. (ed.) Crack dynamics in metallic materials, SpringerVerlag, Vienna, pp. 428-445.

[2] Follansbee, P. S. and Kocks, U. F. (1988), "A constitutive description of the deformation of copper based on the use of the mechanical threshold stress as an internal state variable", Acta Metallurgica, vol. 36, no. 1, pp. 81-93.

[3] Chen, S. and Gray, G. (1996), "Constitutive behavior of tantalum and tantalum-tungsten alloys", Metallurgical and Materials Transactions A, vol. 27, no. 10, pp. 2994-3006.

[4] Goto, D. M., Bingert, J. F., Chen, S. R., Gray III, G. T. and Garrett Jr., R. K. (2000), "The mechanical threshold stress constitutive-strength model description of HY-100 steel", Metallurgical and Materials Transactions A: Physical Metallurgy and Materials Science, vol. 31, no. 8, pp. 1985-1996.

[5] S.R. Chen, P. J., Maudlin, P. J. and Gray, G. T. (1998), "Mechanical properties and constitutive relations for molybdenum under high rate deformation, Molybdenum and Molybdenum Alloys", in Crowson, A., Chen, E. S., Shields, J. A., et al (eds.) The Minerals, Metals and Materials Society, Warrenddale, PA, pp. 155-172.

[6] Gray, G., Chen, S. and Vecchio, K. (1999), "Influence of grain size on the constitutive response and substructure evolution of MONEL 400", Metallurgical and Materials Transactions A, vol. 30, no. 5, pp. 1235-1247.

[7] Steinberg, D. J. (1991), Equation of state and strength properties of selected materials, UCRL-MA-106439, Lawrence Livermore National Laboratory.

[8] Liu, J., (2004), Dyna3D: A Nonlinear, Explicit, Three-Dimensional Finite Element Code for Solid and Structural Mechanics, University of California, Lawrence Livermore National Laboratory, Livermore, (CA) USA.

[9] Gurson, A. L. (1977), "Continuum Theory of Ductile Rupture by Void Nucleation and Growth: Part 1 - Yield Criteria and Flow Rules for Porous Ductile Media", Journal of Engineering Materials and Technology, Transactions of the ASME, vol. 99 Ser H, no. 1, pp. 2-15.

[10] Tvergaard, V. (1982), "Material failure by void coalescence in localized shear bands", International Journal of Solids and Structures, vol. 18, no. 8, pp. 659-672.

[11] Tvergaard, V. and Needleman, A. (1984), "Analysis of the cup-cone fracture in a round tensile bar", Acta Metallurgica, vol. 32, no. 1, pp. 157-169.

[12] Krajcinovic, D. (1996), Damage mechanics, Elsevier, Amsterdam ; New York. 
[13] Lemaitre, J. (1985), "Continuous Damage Mechanics Model for Ductile Fracture", Journal of Engineering Materials and Technology, Transactions of the ASME, vol. 107, no. 1, pp. 83-89.

[14] Voyiadjis, G. Z. and Deliktas, B. (2000), "A coupled anisotropic damage model for the inelastic response of composite materials", Computer Methods in Applied Mechanics and Engineering, vol. 183, no. 3-4, pp. 159-199.

[15] Hammi, Y. (2000), Simulation Numerique de l'Endommagement dans les Precedes de Mise en Forme.

[16] Saanouni, K. and Chaboche, J. L. (2003), "Computational Damage Mechanics. Application to Metal Forming", in de Borst, R. and and Mang, H. A. (eds.) Numerical and Computational Methods ed, Elsevier, Oxford, UK, pp. 321-376.

[17] Zhu, Y. Y. and Cescotto, S. (1995), "A fully coupled elasto-visco-plastic damage theory for anisotropic materials", International Journal of Solids and Structures, vol. 32, no. 11, pp. 1607-1641.

[18] Chow, C. L. and Wang, J. (1987), "An anisotropic theory of elasticity for continuum damage mechanics", International Journal of Fracture, vol. 33, no. 1, pp. 3-16.

[19] Simo, J. C. and Ju, J. W. (1987), "Strain- and stress-based continuum damage modelsI. Formulation", International Journal of Solids and Structures, vol. 23, no. 7, pp. 821840 .

[20] Simo, J. C. and Ju, J. W. (1987), "Strain- and stress-based continuum damage modelsII. Computational aspects", International Journal of Solids and Structures, vol. 23, no. 7, pp. 841-869.

[21] Voyiadjis, G. Z. and Abed, F. H. (2006), "A coupled temperature and strain rate dependent yield function for dynamic deformations of bcc metals", International Journal of Plasticity, vol. 22, no. 8, pp. 1398-1431.

[22] Voyiadjis, G. Z. and Abed, F. H. (2005), "Microstructural based models for bcc and fcc metals with temperature and strain rate dependency", Mechanics of Materials, vol. 37, no. 2-3, pp. 355-378.

[23] Preston, D. L., Tonks, D. L. and Wallace, D. C. (2003), "Model of plastic deformation for extreme loading conditions", Journal of Applied Physics, vol. 93, no. 1, pp. 211-220.

[24] Banerjee, B. and Bhawalkar, A. S. (2008), "An extended mechanical threshold stress plasticity model: Modeling 6061-T9 aluminum alloy", Journal of Mechanics of Materials and Structures, vol. 3, no. 3, pp. 391-424.

[25] Truesdell, C. (1960), Principle of Classical Mechanics and Field Theory Strömungsmechanik III, Springer-Verlag, Berlin.

[26] Noll, W and Truesdell, C, (1965.), The non-linear field theories of mechanics, Springer-Verlag, Berlin; New York. 
[27] Hansen, N. R. and Schreyer, H. L. (1994), "A thermodynamically consistent framework for theories of elastoplasticity coupled with damage", International Journal of Solids and Structures, vol. 31, no. 3, pp. 359-389.

[28] Lubarda, V. A. and Krajcinovic, D. (1995), "Some fundamental issues in rate theory of damage-elastoplasticity", International Journal of Plasticity, vol. 11, no. 7, pp. 763-797.

[29] Rosakis, P., Rosakis, A. J., Ravichandran, G. and Hodowany, J. (2000), "Thermodynamic internal variable model for the partition of plastic work into heat and stored energy in metals", Journal of the Mechanics and Physics of Solids, vol. 48, no. 3, pp. 581-607.

[30] Saanouni, K., Forster, C. and Ben Hatira, F. (1994), "On the anelastic flow with damage", International Journal of Damage Mechanics, vol. 3, pp. 140-169.

[31] Malvern, L. E. (1969), Introduction to the mechanics of a continuous medium, Prentice-Hall, Englewood Cliffs ; London.

[32] Bammann, D. J. (1990), "Modeling Temperature and Strain Rate Dependent Large Deformations of Metals", Applied Mechanics Reviews, vol. 43, no. 5S, pp. S312-S319.

[33] Bammann, D. J., Johnson, J. C. and Chiesa, M. L. (1990), A Strain Rate Dependent Flow Surface Model of Plasticity, SAND90-8227, Sandia National Laboratories.

[34] Cordebois, J. P. and Sidoroff, F. (1980), "Anisotropic Damage in Elasticity and Plasticity.", Journal de mecanique theorique et appliquee, , pp. 45-59.

[35] Kachanov, L. M. (1958), "Time of the rupture process under creep conditions", Ivz Akad Nauk SSR Otd Tech Nauk, vol. 8, pp. 26-31.

[36] Carol, I., Rizzi, E. and Willam, K. (2001), "On the formulation of anisotropic elastic degradation. I. Theory based on a pseudo-logarithmic damage tensor rate", International Journal of Solids and Structures, vol. 38, no. 4, pp. 491-518.

[37] Hill, R., (1950), The mathematical theory of plasticity, Clarendon Press, Oxford.

[38] Tuler, F. R. and Butcher, B. M. (1968), "A criterion for the time dependence of dynamic fracture", International Journal of Fracture Mechanics, vol. 4, no. 4, pp. 431437.

[39] Gilman, J. J. and Tuler, F. R. (1970), "Dynamic fracture by spallation in metals", International Journal of Fracture Mechanics, vol. 6, no. 2, pp. 169-182.

[40] Zhurkov, S. N. (1984), "Kinetic concept of the strength of solids", International Journal of Fracture, vol. 26, no. 4, pp. 295-307.

[41] Meyers, M. A. (1994), Dynamic Behaviour of Materials, John Waley \& Sons, Inc., New York. 
[42] Krausz, A. S. and Eyring, H. (1975), Deformation kinetics, Wiley-Interscience, New York ; London.

[43] Dremin, A. N. and Molodets, A. M. (1985), "On the spall strength of metals", Beijing, Pergamon Press, pp. 13.

[44] Chevrier, P. and Klepaczko, J. R. (1999), "Spall fracture: Mechanical and microstructural aspects", Engineering Fracture Mechanics, vol. 63, no. 3, pp. 273-294.

[45] Hanim, S. and Klepaczko, J. R. (1999), "Numerical study of spalling in an aluminum alloy 7020-T6", International Journal of Impact Engineering, vol. 22, no. 7, pp. 649-673.

[46] Binning, M. S. and Partridge, P. G. (1984), "Subzero tensile properties of 7010 aluminium alloy and Ti-6A1-4V and IMI550 titanium alloys in sheet form", Cryogenics, vol. 24, no. 2, pp. 97-105.

\section{Appendix A}

The damage parameter given in the equations (54) and/or (69) are obtained starting from the general expression for damage accumulation, proposed by Dremin and Molodets [43] (equations (48)):

$$
\phi=\phi_{0} \exp \left(-\frac{\Delta U}{k \theta}\right) \quad 1=\int_{0}^{t_{f}} \phi d t
$$

where the stress dependent activation energy $\Delta U$ is defined following the Yokobori:

$$
\Delta U(\sigma)=\Delta U_{0} \ln \left(\frac{\sigma_{0}}{\sigma}\right)
$$

where $\sigma_{0}$ is the threshold stress which corresponds to the critical time $t_{c 0}$. By substituting expression (A2) into (A1), Klepaczko defined a criterion as:

$$
1=\int_{0}^{t_{f}} \phi d t=\int_{0}^{t_{f}} \phi_{0} \exp \left(-\frac{\Delta U_{0} \ln \left(\frac{\sigma_{0}}{\sigma}\right)}{k \theta}\right) d t=\int_{0}^{t_{f}} \frac{1}{t_{c 0}} \exp \left(\ln \left(\frac{\sigma}{\sigma_{0}}\right)^{\frac{\Delta U_{0}}{k \theta}}\right) d t
$$

or 
$t_{c 0}=\int_{0}^{t_{f}}\left(\frac{\sigma}{\sigma_{0}}\right)^{\frac{\Delta U_{0}}{k \theta}} d t=\int_{0}^{t_{f}}\left(\frac{\sigma}{\sigma_{0}}\right)^{\alpha\left(\Delta U_{0}, \theta\right)} d t$

where $\sigma=\sigma(t)$ and $\phi_{0}=\frac{1}{t_{c 0}}$. Temperature effects in the threshold stress $\sigma_{0}$ are included by using an expression proposed in [45]:

$\sigma_{0}(\theta)=\sigma_{0}^{0} \frac{\mu(\theta)}{\mu_{0}}$

The activation energy is expressed in terms of normalised activation energy $u_{0}$, which provides consistency of the model with the MTS model:

$u_{0}=\frac{\Delta U_{0}}{\mu(\theta) b^{3}}$

By using the expression (A5) for threshold stress and activation energy $\Delta U_{0}$ from (A6), one can rewrite the equation (A4) as:

$t_{c 0}=\int_{0}^{t_{f}}\left(\frac{\sigma(t)}{\sigma_{0}^{0} \frac{\mu(\theta)}{\mu_{0}}}\right)^{\frac{\mu(\theta) b^{3} u_{0}}{k \theta}} d t=\int_{0}^{t_{f}}\left(\frac{\sigma(t) / \mu(\theta)}{\sigma_{0}^{0} / \mu_{0}}\right)^{\frac{\mu(\theta) b^{3} u_{0}}{k T}} d t$

where the stress is a function of time. Having defined the (A7), damage parameter for a reference direction is defined in time domain as:

$\beta_{i}(t)=\frac{\int_{0}^{t_{f}}\left(\frac{\sigma_{i}(t) / \mu(\theta)}{\sigma_{0}^{0} / \mu_{0}}\right)^{\frac{\mu(\theta) b^{3} u_{0}}{k \theta}} d t}{t_{c 0}}$

or as a function of strain for the constant strain rate as:

$\beta_{i}(\varepsilon)=\frac{\int_{0}^{\varepsilon_{f}}\left(\frac{\sigma_{i}(\varepsilon) / \mu(\theta)}{\left.\sigma_{0}^{0} / \mu_{0}\right)^{\frac{\mu(\theta) b^{3} u_{0}}{k \theta}} d \varepsilon}\right.}{\varepsilon_{c 0}}$ 
For the sake of material characterisation of the damage model, the equation (A8) was expressed in terms of strain and strain rate using the following rules: $t=\varepsilon / \varepsilon$ and $t_{c 0}=\varepsilon_{c 0} / \varepsilon$. Provided that stress strain relationship is linear, the damage parameter after integration is obtained as:

$D_{i}(\varepsilon)=\frac{\varepsilon}{\varepsilon_{c 0}} \frac{1}{\left(\frac{\mu(\theta) b^{3} u_{0}}{k T}+1\right)}\left(\frac{\sigma_{i}(\varepsilon) / \mu(\theta)}{\sigma_{0}^{0} / \mu_{0}}\right)^{\frac{\mu(\theta) b^{3} u_{0}}{k \theta}}$

\section{Appendix B}

Taylor anvil test is routinely used in validation of constitutive models. It is attractive because it is characterised by a wide range of plastic strains, strain rates and temperatures. The test that was performed (impact velocity $200 \mathrm{~m} / \mathrm{s}$ ) was designed to generate strain rates up to $\varepsilon=6 \times 10^{3} s^{-1}$ as illustrated in the figures from Figure 18 to Figure 20 . The strain rate histories in the impact direction were recorded in two cross sections of the specimen. The curve denoted D5 in the Figure 18 corresponds to an element in the cylinder-anvil contact plane. The other two curves (B736 and C740) were recorded in the cross section at distance of $10 \mathrm{~mm}$ from the contact plane. The contour plots of the strain rate in the impact and in plane directions in the two time instances are shown in Figure 19 and Figure 20, respectively. For the sake of completeness, the stress distribution in the $\mathrm{Z}$ direction (impact direction) in these two time instances are illustrated Figure 21.

\section{Near Figure 18}

Near Figure 19 
Near Figure 20

Near Figure 21 




Figure 1 The MTS model approximations vs. experimental data for range of temperatures and strain rates

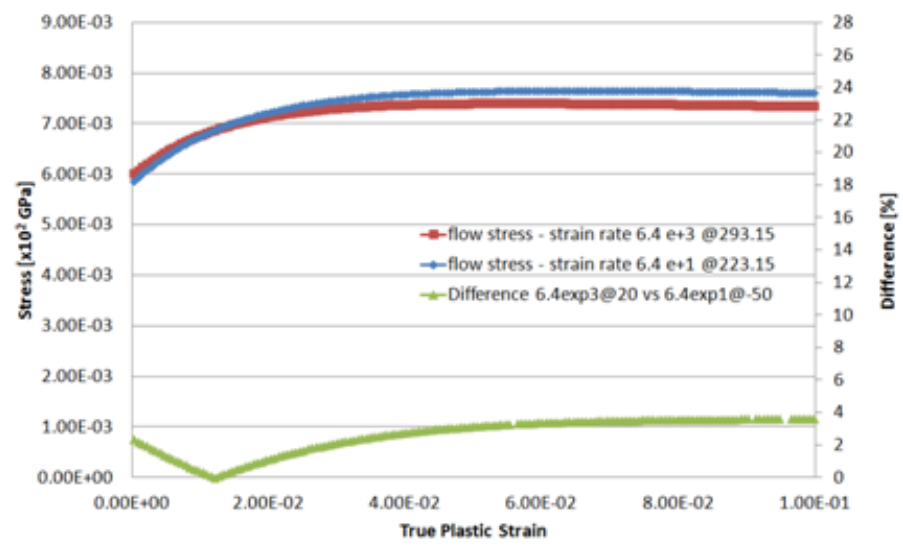

Figure 2 The MTS flow stress - plastic strain curves for two loading cases: the high loading rate $\varepsilon=6.4 \times 10^{3} \mathrm{~s}^{-1}$ at $\theta=293.15 \mathrm{~K}$; and the loading rate $\varepsilon=64 \mathrm{~s}^{-1}$ at $\theta=223.15 \mathrm{~K}$



Figure 3 Equating damage work [17] 




Figure 4 Damage variable $D_{1}$ vs. strain under simple tension for AA7010

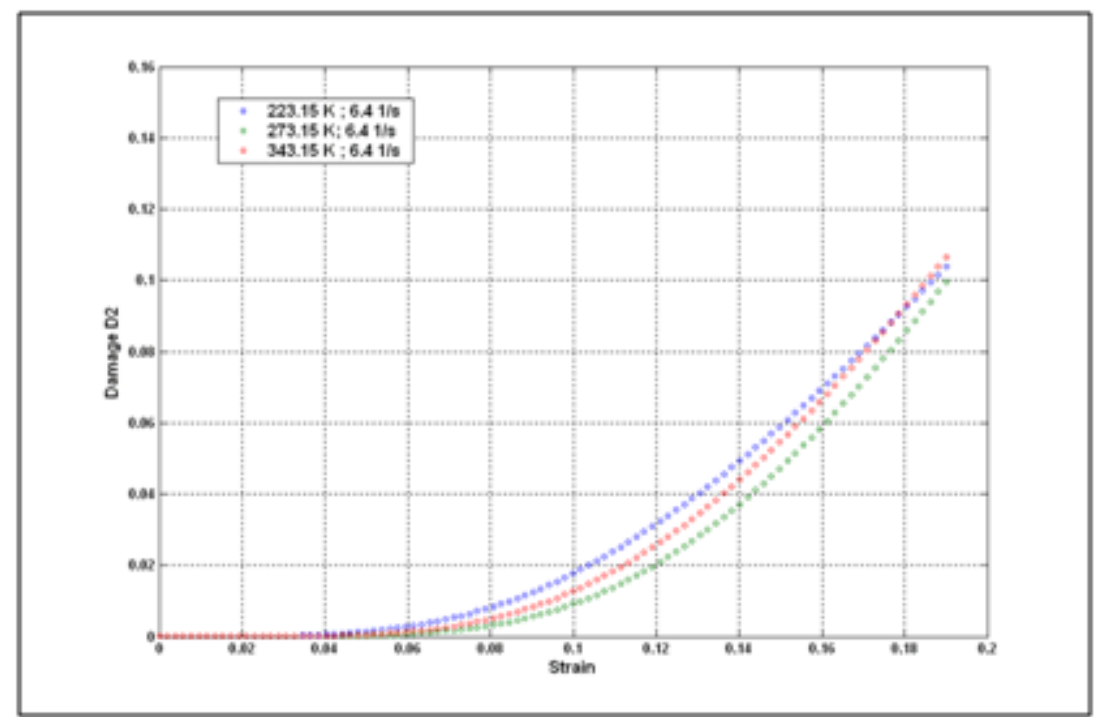

Figure 5 Damage variable $D_{2}$ vs. strain under simple tension of AA7010 


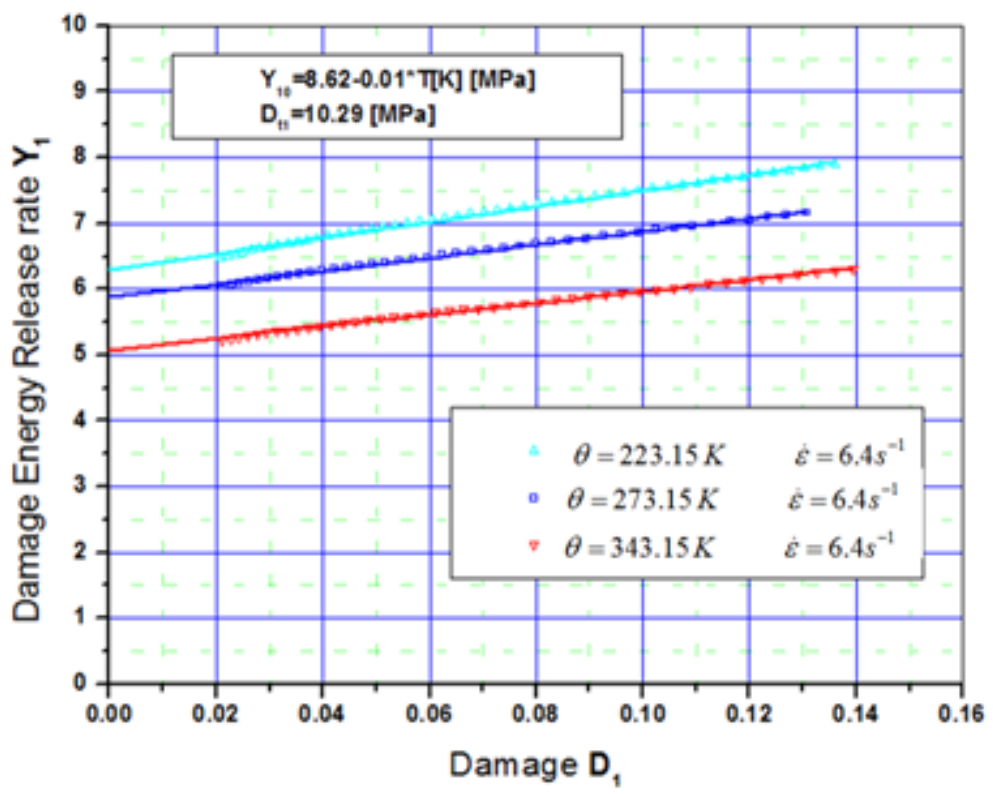

Figure 6 The damage energy density released rate vs. damage for AA7010 at $\varepsilon=6.4 \mathrm{~s}^{-1}$

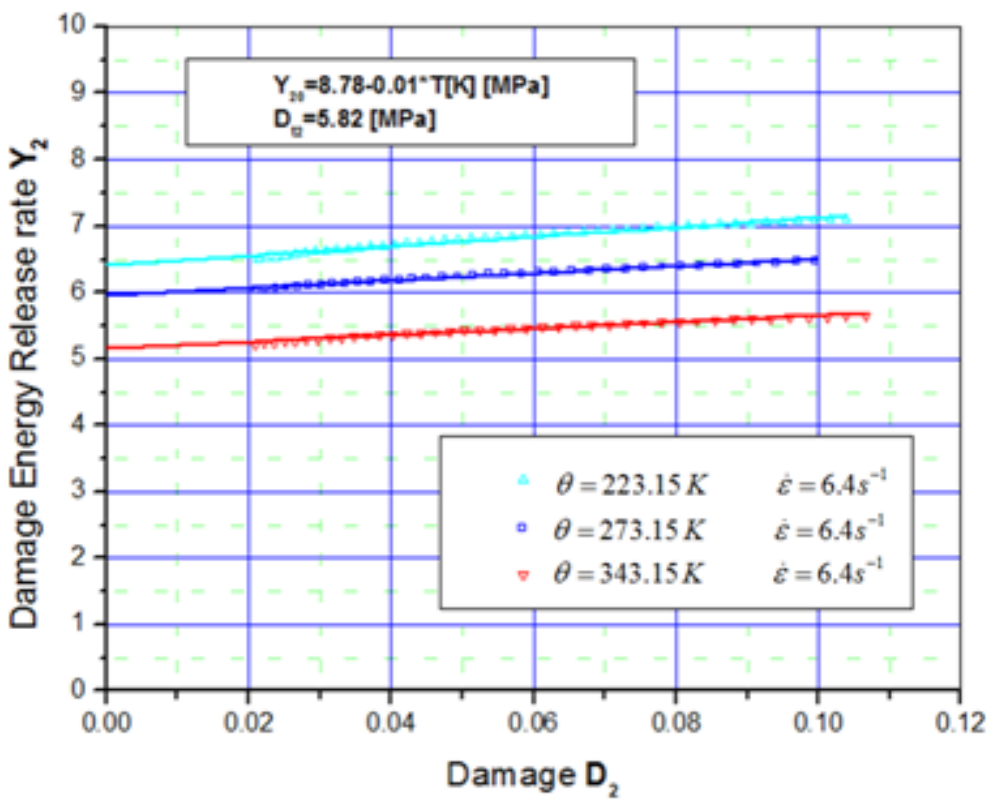

Figure 7 The damage energy density released rate vs. damage for AA7010 at $\varepsilon=6.4 \mathrm{~s}^{-1}$ 


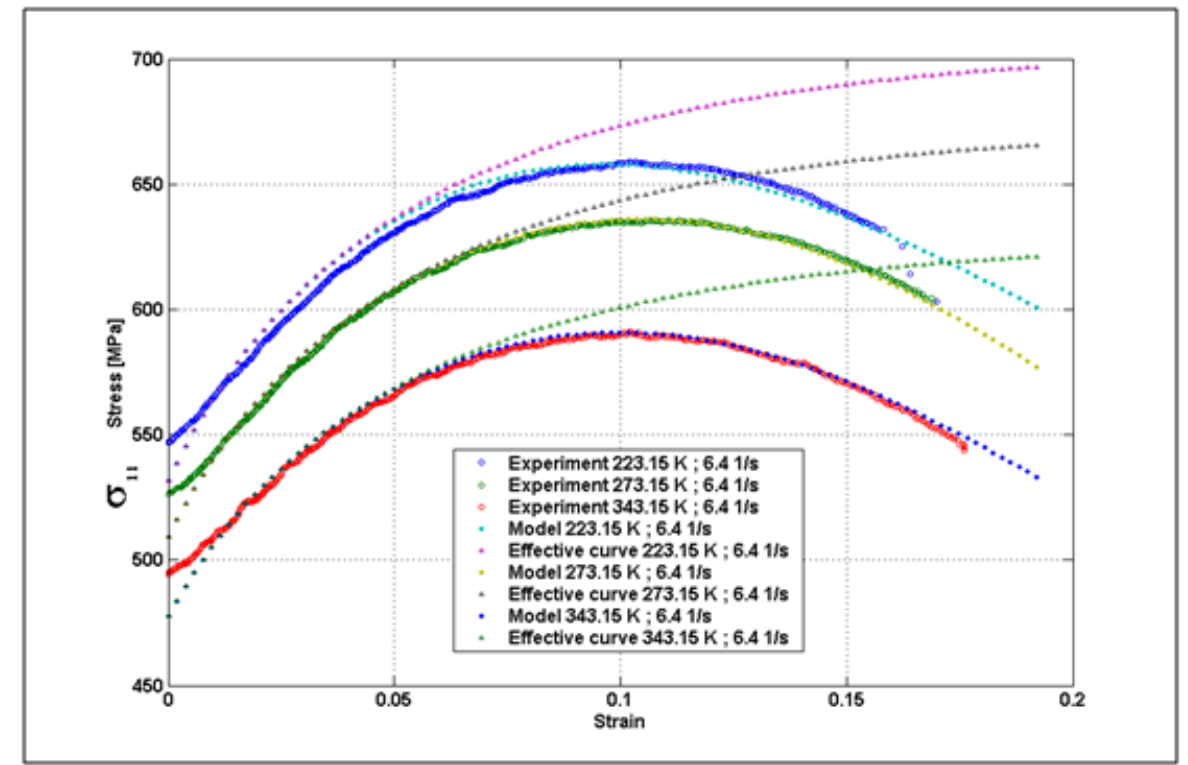

Figure $8\left(\sigma_{11} \varepsilon_{11}\right)$ experimental, effective (MTS) and model (MTS+damage) stress-strain curves of AA7010 at $\varepsilon=6.4 s^{-1}$



Figure $9\left(\sigma_{22} \varepsilon_{22}\right)$ Calculated stress-strain curves of AA7010 at $\varepsilon=6.4 \mathrm{~s}^{-1}$ 


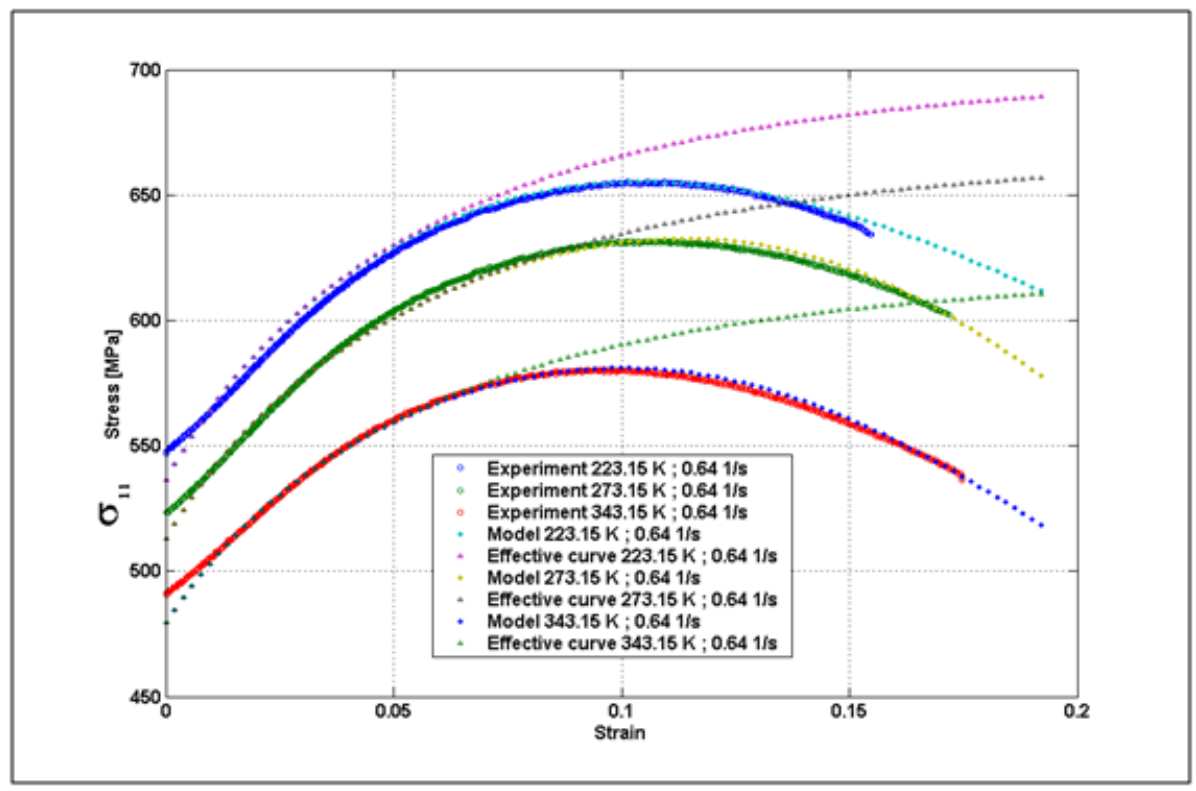

Figure $10\left(\sigma_{11} \varepsilon_{11}\right)$ experimental, effective (MTS) and model (MTS+damage) stress-strain curves of AA7010 at $\varepsilon=6.4 \times 10^{-1} s^{-1}$

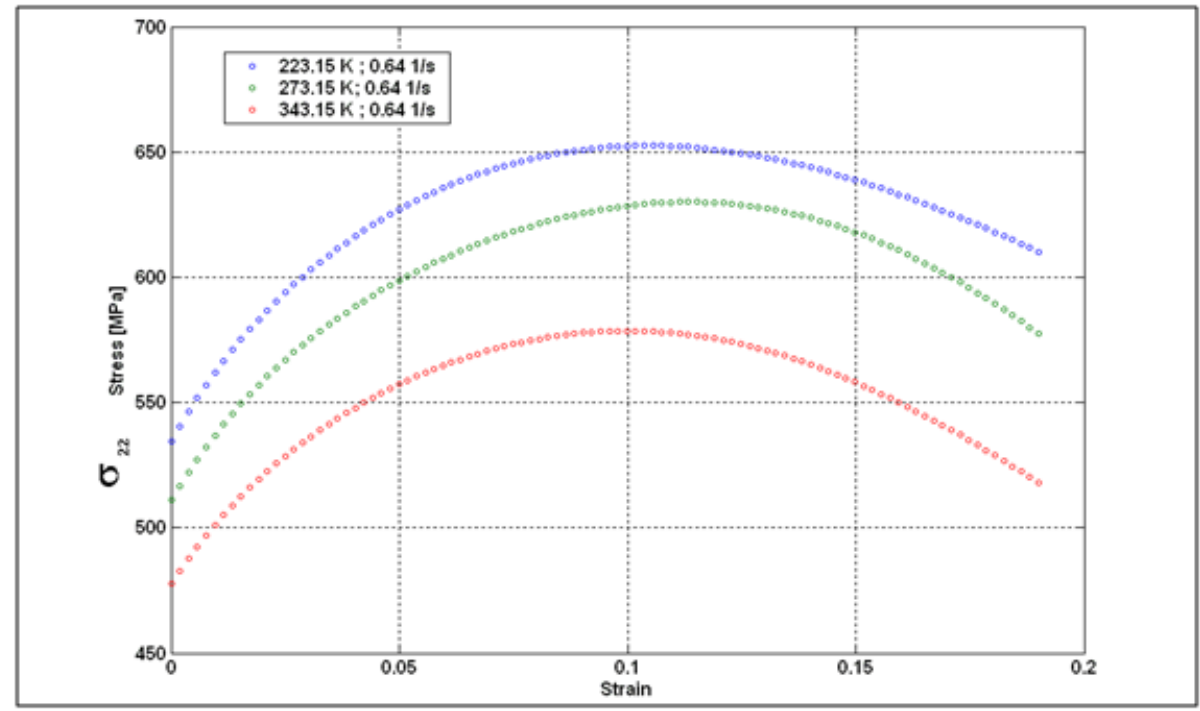

Figure $11\left(\sigma_{22} \varepsilon_{22}\right)$ Calculated stress-strain curves of AA7010 at $\varepsilon=6.4 \times 10^{-1} \mathrm{~s}^{-1}$

Figure 12 Schematic drawing of a Taylor cylinder impact test 



Figure 13 Photographs of the post-test geometry for the AA 7010 Taylor specimen after impact at $200 \mathrm{~m} / \mathrm{s}$


Figure 14 Scan of post-test geometry and digitized foot print for the AA 7010 Taylor specimen



Figure 15 Comparisons of the major and minor side profiles of post-test geometry for the AA 7010 Taylor specimens impacted at $200 \mathrm{~m} / \mathrm{s}$ plotted as radial strain vs. distance 


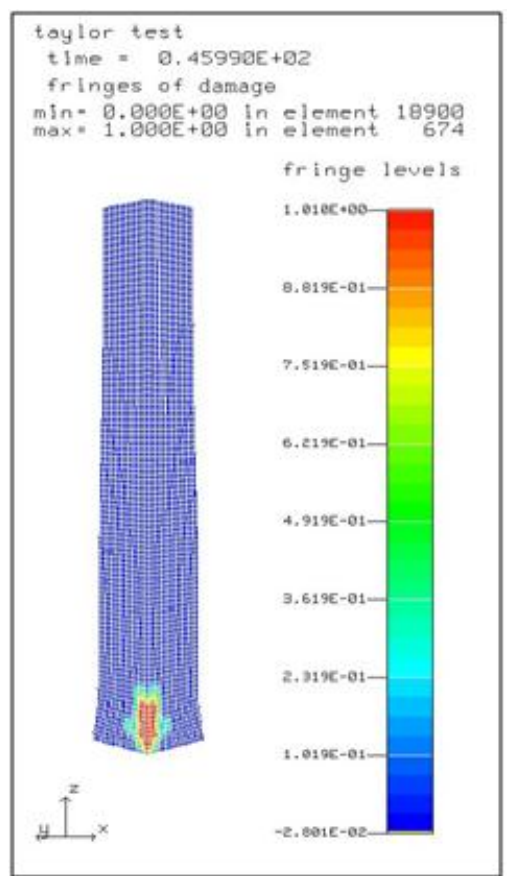

Figure 16 Damage fields generated in AA7010 Taylor specimen



Figure 17 Comparison of experimental and simulation results for major and minor distributions of plastic strains of Taylor cylinder test impacted at $200 \mathrm{~m} / \mathrm{s}$

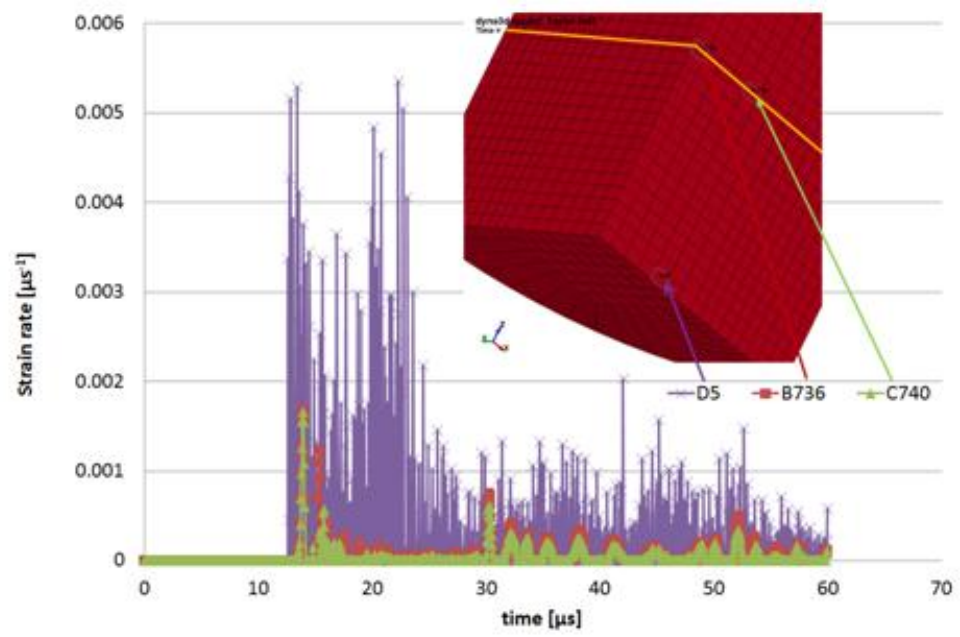

Figure $18 \varepsilon_{z}$-strain rate history in two cross sections (impact plane and at distance of $10 \mathrm{~mm}$ from the impact plane) 

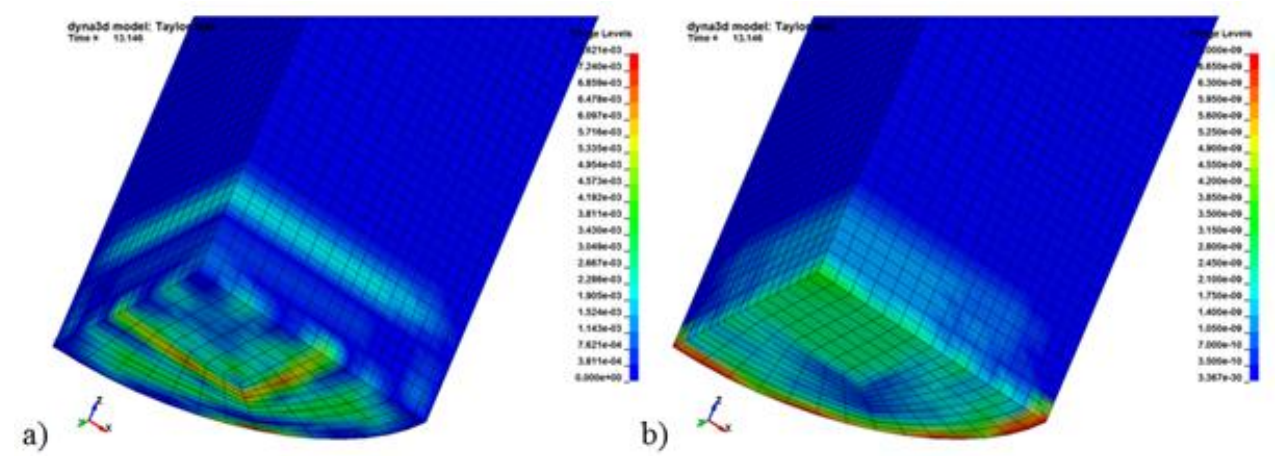

Figure 19 Taylor anvil test - distribution of the strain rate $0.5 \mu$ s after impact a) strain rate $\varepsilon_{z}$ (impact direction); b) strain rate $\varepsilon_{y}$ (in plane direction)

a)

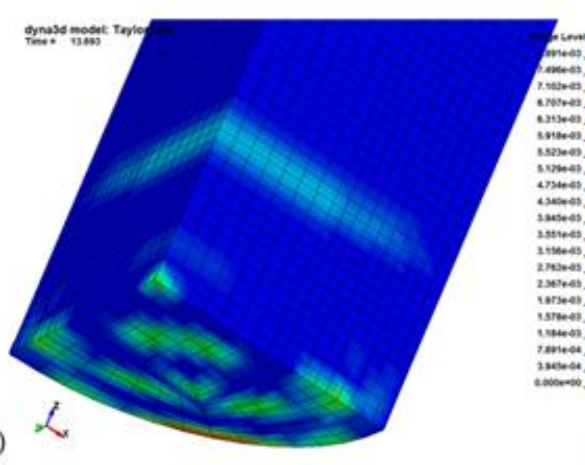

b)



Figure 20 Taylor anvil test - distribution of the strain rate $1 \mu$ s after impact: strain rate $\varepsilon_{z}$ (impact direction); b) strain rate $\varepsilon_{y}$ (in plane direction)

a)



b)

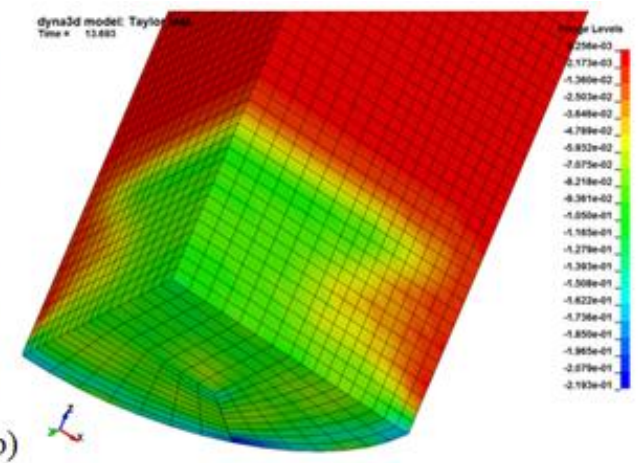

Figure 21 Taylor anvil test $-\sigma_{z}$ stress distribution (impact direction): a) $0.5 \mu$ s after the impact and b) $1 \mu$ s after the impact 